\title{
Beam Tracking for Interference Alignment in Slowly Fading MIMO Interference Channels: A Perturbations Approach Under a Linear Framework
}

\author{
Heejung Yu, Student Member, IEEE, Youngchul Sung, Senior Member, IEEE, Haksoo Kim, Student Member, IEEE, \\ and Yong H. Lee, Senior Member, IEEE
}

\begin{abstract}
In this paper, the beam design for signal-space interference alignment in slowly fading multiuser multiple-input multiple-output (MIMO) interference channels is considered. Based on a linear formulation for interference alignment, a predictive beam tracking algorithm is proposed using matrix perturbation theory. The proposed algorithm, based on a mixture of iteration and update, computes interference-aligning beamforming vectors at the current time by updating the previous beam vectors based on the channel difference between the two time steps during the predictively updating phase, and yields significant reduction in computational complexity compared with existing methods recalculating beams at each time step. The tracking performance of the algorithm is analyzed in terms of mean square error and sum rate loss between the predictively updating approach and the recalculating approach, and the impact of imperfect channel knowledge is also investigated under the state-space channel model. Numerical results show that the proposed algorithm has almost the same performance as non-predictive methods in sum rate. Thus, the proposed algorithm provides a very efficient way to realize interference alignment in a realistic slowly fading MIMO channel environment.
\end{abstract}

Index Terms-Interference alignment, least squares, null space tracking, perturbation theory, predictive algorithm, slowly fading channels.

\section{INTRODUCTION}

$\mathbf{I}$ NTERFERENCE alignment is one of the promising techniques to handle interference properly in a future interference-limited wireless environment. Interference alignment achieves the upper bound of the degrees of freedom (DoF) in multiuser interference channels by confining the interference from all undesired transmitters into an interference subspace at each receiver [1], and with the technique, each user can approximately achieve a half of the capacity that can be achieved

Manuscript received March 03, 2011; revised August 09, 2011 and November 22, 2011; accepted December 18, 2011. Date of publication December 23, 2011; date of current version March 06, 2012. The associate editor coordinating the review of this manuscript and approving it for publication was Dr. Josep Vidal.

This work was supported by the IT R\&D program of MKE/KEIT. (2008-F004-02, "5G mobile communication systems based on beam division multiple access and relays with group cooperation").

The authors are with the Department of Electrical Engineering, KAIST, Daejeon, 305-701, Republic of Korea (e-mail: heejung@etri.re.kr; ysung@ee. kaist.ac.kr; ; hskim@stein.kaist.ac.kr; yohlee@ee.kaist.ac.kr).

Color versions of one or more of the figures in this paper are available online at http://ieeexplore.ieee.org.

Digital Object Identifier 10.1109/TSP.2011.2181502 without interference. Thus, the interference alignment technique is useful when wireless networks are interference-limited with a high signal-to-noise ratio (SNR) due to the small cell size. One line of research in this area has focused on the DoF characterization and feasibility of exact alignment from information-theoretical perspectives [1]-[7]. Such research is important since it provides the fundamental limit of the scheme and insights into developing good algorithms. Along with this, the other line of research is on the invention of efficient beam design algorithms for interference alignment to realize the potential of the technique from signal processing perspectives [8]-[10]. Among several known schemes for interference alignment, the signal-space alignment based on the single-shot MIMO technique [1], i.e., beamforming using multiple antennas, is most attractive from a practical viewpoint, and this scheme is the main focus of this paper. To illustrate the necessity of efficient algorithms in this case as well as in other schemes, consider off-line beam design for interference alignment using a look-up table. In this case, sets of beam vectors are calculated for different channel realizations beforehand and stored in a table, and a particular set is selected based on channel information. Even in a simple case of 3 -user $2 \times 2$ MIMO channel, this scheme requires a memory space of a 72 bit address even with one bit assigned for one real channel coefficient $\left(2 M^{2} K^{2}=72\right.$, where the factor of 2 is for real and imaginary components of a channel coefficient, and $K$ and $M$ are the numbers of users and antennas, respectively). Thus, such a scheme is impractical, and fast real-time algorithms are necessary so that the technique can be used in practice. This is especially true for time-varying channels in a practical wireless environment since interference-aligning beam vectors should be computed at every time step.

\section{A. Our Approach and Summary of Result}

In this paper, we consider the beam design problem in multiuser slowly fading MIMO interference channels, where beam vectors need to be computed at every time step. The assumption of slow fading is suitable for realistic wireless environments where the channels at two consecutive time steps are not independent and the current channel is a slightly updated version of the previous one. One could apply a beam design method for interference alignment invented under the assumption of a time-invariant MIMO channel to every time step with different channel state information (CSI) even in this time-varying case. However, this may not be an efficient way since this approach 
does not exploit the time dependency of the channels between the consecutive time steps. One would expect that the beam solution at the current time is a slightly updated version of that at the previous time due to the channel's time dependency. Such updating or recursive approaches have played a major role in inventing efficient algorithms for important estimation problems like the deterministic least squares and minimum mean square error (MMSE) estimation and have resulted in the celebrated recursive least squares (RLS) algorithm and Kalman filtering. As evident in these two examples, updating or recursive solutions require some key ingredient that makes them possible, e.g., the matrix inversion lemma for RLS and the state-space model for Kalman filtering. This is also true in the considered case of beam tracking for interference alignment in slowly fading MIMO channels.

Our approach to an updating solution to this problem is based on our previous work of linear formulation for interference alignment, which is unlike existing bilinear formulations and which converts the problem of interference alignment to solving a single linear system with dummy variables [9], [11]. Applying matrix perturbation theory to this linear formulation, we here develop a beam tracking algorithm for interference alignment in slowly fading MIMO interference channels. Under the linear formulation, an interference-aligning beam solution is given by the eigenvector associated with the smallest eigenvalue of the misalignment covariance matrix (which will be defined later), and the solution to a perturbed misalignment covariance matrix by channel variation can be obtained by subspace tracking. The proposed algorithm computes interference-aligning beam vectors at the current time by additively updating those of the previous time, and reduces computational complexity significantly compared with existing batch methods with which interference-aligning beam vectors are redesigned at every time step when applied to time-varying MIMO interference channels. In addition to the development of a tracking algorithm for interference alignment, as a byproduct we also derive a new update formula for general null space tracking that is similar to the existing result in [12] and is useful in other applications too.

The tracking performance of the algorithm is analyzed in terms of mean square error (MSE) and sum data rate. The tracking error results from two sources: predictive beam design and imperfect channel knowledge due to channel estimation. The error by prediction is evaluated analytically with a perturbations approach, and the tracking error caused by imperfect channel knowledge is examined under the state-space channel model and time-division duplex (TDD) transmission structure. Numerical results show that the proposed predictive algorithm tracks the time-varying beam solution well for a wide range of fading rate or mobile speed with significantly reduced complexity.

\section{B. Related Works}

The interference alignment was introduced for MIMO $X$-channels [13], [14]. It was shown that the interference alignment achieved the maximum DoF in $K$-user single-input single-output (SISO) time-varying interference channels based on a vector channel model induced by time concatenation [1]. In the case of time-invariant $M \times M$ MIMO channels (a single-shot approach), it was shown that the maximum $3 \frac{M}{2}$ DoF could be achieved with a beamforming approach in the 3 -user case [1]. It was also shown for $K \geq 4$ that exact interference alignment with a signal space approach was infeasible in time-invariant MIMO channels. Also, interference alignment based on a signal scale using structured coding has been proposed for deterministic channel models [15], [16]. With asymmetric complex signaling, $1.2 \mathrm{DoF}$ can be achieved for $K \geq 3$ for almost all values of channels [17]. It has been shown that the total DoF is determined by less than $\frac{K}{2}$ in the fully connected constant real Gaussian interference channels depending on the rationality and irrationality of channel coefficients [3]. The total DoF of $\frac{K}{2}$ is achieved for the set of measure zero of channel gains. Recently, the achievability of $\frac{K M}{2}$ DoF using a new interference alignment based on the Khinchin-Groshev theorem has been proved [4] by exploiting the properties of rationally independent channel coefficients. Based on this result, interference alignment for MIMO interference channels was proposed in [6]. In [7], the relay-aided interference alignment including achievable DoF, feasibility, and algorithm was discussed. The feasibility conditions for interference alignment under different frameworks were derived in [5] and [18].

In addition to these information-theoretical results, there has been research from algorithmic aspects. Gomadam et al. proposed algorithms to minimize interference and maximize the signal-to-interference-plus-noise ratio (SINR) by solving a system of bilinear equations iteratively [8]. A linear formulation to interference alignment using a beamforming technique for time-invariant MIMO channels was introduced, and iterative algorithms based on least squares were proposed in [9]. Recent advances for interference alignment include the investigation of channel uncertainty and SINR characterization [19], [20], limited feedback in single and multiantenna channels [21], [22], interference alignment scheme based on alternating minimization [10], and ergodic interference alignment using the statistical properties of channels [23]. Additionally, interference alignment without channel information at transmitters was proposed in [24].

\section{Notations and Organization}

We will make use of standard notational conventions. Vectors and matrices are written in boldface with matrices in capitals. All vectors are column vectors. For a matrix $\mathbf{A}, \mathbf{A}^{T}$ and $\mathbf{A}^{H}$ indicate the transpose and Hermitian transpose of $\mathbf{A}$, respectively, and $\operatorname{vec}(\mathbf{A})$ is the column vector consisting of all the columns of $\mathbf{A} . \mathcal{C}(\mathbf{A})$ represents the column space of $\mathbf{A}$, i.e., the linear subspace spanned by the columns of $\mathbf{A} . \mathbf{A}=\left[a_{i j}\right]$ means that $\mathbf{A}$ is a matrix composed of $a_{i j}$ denoting the $i$ th row and $j$ th column element (also sometimes denoted as $[\mathbf{A}]_{i j}$ ), and $\operatorname{diag}\left(d_{1}, \ldots, d_{n}\right)$ is the diagonal matrix of diagonal elements $d_{1}, \ldots, d_{n} . \mathbf{A}^{\dagger}$ and $\operatorname{tr}(\mathbf{A})$ denote the pseudo inverse and trace of matrix $\mathbf{A}$, respectively. We use $\|\mathbf{a}\|$ for the 2-norm of a vector a, $\|\mathbf{A}\|_{F}$ and $\|\mathbf{A}\|_{2}$ denote the Frobenius norm and 2-norm of a matrix $\mathbf{A}$, respectively. For matrices $\mathbf{A}$ and $\mathbf{B}, \mathbf{A} \otimes \mathbf{B}$ and $\mathbf{A} \circ \mathbf{B}$ denote the Kronecker and Hadamard products between the two matrices, respectively. $\mathbf{I}_{n}$ stands for the identity matrix of size $n \times n$ (the subscript is omitted when unnecessary), and $\mathbf{1}$ is a column vector with all elements as one. $\mathbf{0}$ is an all-zero 
matrix. The notation $\mathbf{x} \sim \mathcal{C} \mathcal{N}(\boldsymbol{\mu}, \boldsymbol{\Sigma})$ means that $\mathbf{x}$ is complex Gaussian distributed with mean vector $\boldsymbol{\mu}$ and covariance matrix $\Sigma$. $\mathbb{E}\{X\}$ denotes the expectation of random variable $X$. For a scalar $a, a^{*}$ is the complex conjugate of $a$.

The paper is organized as follows. The system model and previous work on a linear framework approach to interference alignment are described in Section II. In Section III, a predictive beam design algorithm for interference alignment is proposed based on matrix perturbation theory by exploiting our linear framework. In Section IV, the performance with consideration of channel prediction and the computational complexity of the proposed algorithm are examined. Numerical results are provided in Section V, followed by the conclusion in Section VI.

\section{System Model AND PRELIMINARIES}

We consider a $K$-user $M \times N$ MIMO interference channel where the $K$ transmitter-receiver pairs exist with interfering links. We assume that each transmitter and receiver have $N$ and $M$ antennas, respectively, and $M \leq N$. We also assume that the MIMO channel is flat-fading. Due to the interference from other transmitters, the received signal at receiver $k$ is given by

$$
\begin{aligned}
\mathbf{y}_{k}[n]=\mathbf{H}_{k k}[n] \mathbf{V}_{k}[n] \mathbf{s}_{k}[n] & \\
& +\sum_{l=1, l \neq k}^{K} \mathbf{H}_{k l}[n] \mathbf{V}_{l}[n] \mathbf{s}_{l}[n]+\mathbf{n}_{k}[n]
\end{aligned}
$$

where $n$ is the time index, $\mathbf{H}_{k l}[n]$ is the $M \times N$ MIMO channel matrix between transmitter $l$ and receiver $k$, $\mathbf{V}_{l}[n]=\left[\mathbf{v}_{1}^{(l)}[n], \ldots, \mathbf{v}_{d_{l}}^{(l)}[n]\right]$ is an $N \times d_{l}$ beamforming matrix at transmitter $l, \mathbf{s}_{l}[n]=\left[s_{1}^{(l)}[n], \ldots, s_{d_{l}}^{(l)}[n]\right]^{T}$ is the $d_{l} \times 1$ signal vector with unit power, and $\mathbf{n}_{k}[n] \sim \mathcal{C N}\left(0, \sigma^{2} \mathbf{I}\right)$ is zero-mean complex Gaussian noise vector with covariance matrix $\sigma^{2} \mathbf{I}$. Here, $d_{l}$ is the number of data streams at transmitter $l$. It is assumed that the number of data streams at each transmitter is equal, i.e., $d_{1}=\cdots=d_{K}=d$. We also assume that the MIMO channels $\left\{\mathbf{H}_{k l}[n]\right\}$ are slowly time-varying and that all CSI is available at each transmitter.

\section{A. Preliminaries: Linear Framework for Interference Alignment [9]}

In this subsection, we briefly review the previous work [9] that provides a linear framework for interference alignment when time $n$ is fixed. Under the assumptions of $d_{1}=\cdots=d_{K}=d=\frac{M}{2}$ and $M \leq N$, the interference from all undesired transmitters should be confined to an interference subspace with dimension $\frac{M}{2}$ at each receiver for interference alignment, and the interference alignment condition in this case is given by

$$
\begin{aligned}
& \mathcal{C}\left(\mathbf{H}_{12} \mathbf{V}_{2}\right)= \mathcal{C}\left(\mathbf{H}_{13} \mathbf{V}_{3}\right)=\cdots=\mathcal{C}\left(\mathbf{H}_{1 K} \mathbf{V}_{K}\right) \\
& \mathcal{C}\left(\mathbf{H}_{21} \mathbf{V}_{1}\right)= \mathcal{C}\left(\mathbf{H}_{23} \mathbf{V}_{3}\right)=\cdots=\mathcal{C}\left(\mathbf{H}_{2 K} \mathbf{V}_{K}\right) \\
& \vdots \\
& \mathcal{C}\left(\mathbf{H}_{K 1} \mathbf{V}_{1}\right)=\mathcal{C}\left(\mathbf{H}_{K 2} \mathbf{V}_{2}\right)=\cdots=\mathcal{C}\left(\mathbf{H}_{K K-1} \mathbf{V}_{K-1}\right)
\end{aligned}
$$

(Throughout this section, the time index $n$ is fixed and is dropped for notational simplicity.) The conditions in (2) can be converted into a system of linear equations with dummy variables. Consider the first equality in the first row of (2)

$$
\mathcal{C}\left(\mathbf{H}_{12}\left[\mathbf{v}_{1}^{(2)}, \ldots, \mathbf{v}_{d}^{(2)}\right]\right)=\mathcal{C}\left(\mathbf{H}_{13}\left[\mathbf{v}_{1}^{(3)}, \ldots, \mathbf{v}_{d}^{(3)}\right]\right) .
$$

A necessary and sufficient condition for the equivalence of column spaces of two matrices is that a column in one matrix is represented by a linear combination of the columns of the other. Thus, the subspace equivalence in (3) can be rewritten as the following equation:

$$
\mathbf{H}_{12} \mathbf{v}_{i}^{(2)}=\sum_{j=1}^{d} \alpha_{i j}^{(13)} \mathbf{H}_{13} \mathbf{v}_{j}^{(3)}, \quad i=1, \ldots, d
$$

where $\alpha_{i j}^{(13)}, i, j=1,2, \ldots, d$, are coefficients for linear combination. The multiple equalities in (4) can be written in a single matrix-vector form as

$$
\left(\mathbf{I}_{d} \otimes \mathbf{H}_{12}\right) \operatorname{vec}\left(\mathbf{V}_{2}\right)-\left(\mathbf{A}_{13} \otimes \mathbf{H}_{13}\right) \operatorname{vec}\left(\mathbf{V}_{3}\right)=\mathbf{0}
$$

where $\mathbf{A}_{13}=\left[\alpha_{i j}^{(13)}\right]$. In this manner, each row in (2) can be converted into a linear equation. Collecting all equations generated from the $K$ rows in (2) yields the following system of linear equations with dummy variables $\left\{\mathbf{A}_{k l}\right\}$ :

$$
\tilde{\mathbf{H}} \mathbf{v}=\mathbf{0}
$$

where $\tilde{\mathbf{H}}$ is defined as shown in (7) at the bottom of the next page, and

$$
\mathbf{v} \triangleq\left[\operatorname{vec}\left(\mathbf{V}_{1}\right)^{T}, \operatorname{vec}\left(\mathbf{V}_{2}\right)^{T}, \ldots, \operatorname{vec}\left(\mathbf{V}_{K}\right)^{T}\right]^{T} .
$$

Note here that $\tilde{\mathbf{H}}$ is a function of channel $\left\{\mathbf{H}_{k l}\right\}$ and dummy variables $\left\{\mathbf{A}_{k l}\right\}$. Once the channel and dummy variables are given, we can obtain the interference-aligning beam vector $\mathbf{v}$ from (6). On the other hand, the linear combination coefficients $\left\{\mathbf{A}_{k l}\right\}$ can also be obtained easily when $\left\{\mathbf{V}_{l}\right\}$ are given. Note that (4) can be rewritten as

$$
\mathbf{H}_{12} \mathbf{V}_{2}=\mathbf{H}_{13} \mathbf{V}_{3} \mathbf{A}_{13}^{T}
$$

and, thus, $\mathbf{A}_{13}$ is simply given by

$$
\mathbf{A}_{13}=\left\{\left(\mathbf{H}_{13} \mathbf{V}_{3}\right)^{\dagger} \mathbf{H}_{12} \mathbf{V}_{2}\right\}^{T}
$$

where $\mathbf{H}_{13} \mathbf{V}_{3}$ is always a tall matrix (i.e., the number of rows of the matrix is larger than that of its columns) and, thus, its pseudoinverse almost surely exists because the number of data streams is always less than the number of receiver antennas. [In the case of $d=\frac{M}{2}=1$, the right-hand side (RHS) of (10) reduces simply to taking the inner product between two vectors.] In a similar manner, $\left\{\mathbf{A}_{k l}\right\}$ are given by

$$
\left\{\begin{array}{l}
\mathbf{A}_{1 j}=\left\{\left(\mathbf{H}_{1 j} \mathbf{V}_{j}\right)^{\dagger} \mathbf{H}_{12} \mathbf{V}_{2}\right\}^{T}, j=3, \ldots, K \\
\mathbf{A}_{i j}=\left\{\left(\mathbf{H}_{i j} \mathbf{V}_{j}\right)^{\dagger} \mathbf{H}_{i 1} \mathbf{V}_{1}\right\}^{T}, i, j=2, \ldots, K, j \neq i .
\end{array}\right.
$$

The size of $\tilde{\mathbf{H}}$ is $\frac{K(K-2) M^{2}}{2} \times \frac{K M N}{2}$, and the existence of a nontrivial solution to (6) depends on the system parameters $K, M$, 
and $N$. First, consider the case where exact interference alignment is not feasible ${ }^{1}$. In this case, $\tilde{\mathbf{H}}$ is necessarily a tall matrix, and a nontrivial solution to (6) does not exist for any $\left\{\mathbf{A}_{k l}\right\}$. Thus, in this case we can find the best approximate solution to interference alignment by resorting to a least squares approach

$$
\hat{\mathbf{v}}=\underset{\|\mathbf{v}\|=1}{\arg \min }\|\tilde{\mathbf{H}} \mathbf{v}\|^{2}
$$

and the optimal solution to (6) in the least squares sense can be found by an iterative algorithm in which (11) and (12) are used alternately at each iteration. This algorithm converges and its rate of convergence is much faster than other algorithms for interference alignment with similar performance [9]. One can view $\|\tilde{\mathbf{H}} \mathbf{v}\|^{2}$ as the power of overall interference missalignment, and hereafter we refer to $\tilde{\mathbf{H}}$ as the interference misalignment matrix. It is known that the solution to (12) is the eigenvector of $\tilde{\mathbf{H}}^{H} \tilde{\mathbf{H}}$ corresponding to the smallest eigenvalue. Second, consider the case where exact interference alignment is feasible, and, hence, a nontrivial solution solution for $\mathbf{v}$ in (6) exists. [Note that (12) is still valid in this case.] This case can be divided further into two subcases: one is that $\tilde{\mathbf{H}}$ is still a tall matrix (e.g., $K=3, M=N=2 d$ ) and the other is that $\tilde{\mathbf{H}}$ is a strictly fat matrix (e.g., $K=3, M=2, N=3$ ). In the latter case, an interference-aligning solution $\mathbf{v}$ can be found simply as a null vector of $\tilde{\mathbf{H}}$ with an arbitrary choice of $\left\{\mathbf{A}_{k l}\right\}$ and multiple linearly independent null vectors exist. In the former case, on the other hand, $\tilde{\mathbf{H}}$ becomes rank-deficient only with proper $\left\{\mathbf{A}_{k l}\right\}$, and a nontrivial solution for $\mathbf{v}$ in (6) can be found again by the iterative algorithm based on (11) and (12).

One drawback of the iterative algorithm in [9] is that its performance is degraded when $d>1$ due to the interference from other data streams of the same transmitter. In the next subsection, we show that this difficulty can be overcome by choosing the beamforming vector of each stream from different basis vectors of the null space of $\tilde{\mathbf{H}}$.

\footnotetext{
${ }^{1}$ The feasibility analysis is not the main focus of this paper. A general feasibility study can be found in [5].
}

\section{B. Enhanced Beamforming Vector for $d>1$}

In this subsection, we first discuss details about the reason why the performance of the iterative algorithm in [9] is degraded when $d>1$, and then present a method for constructing enhanced beamforming vectors with a DoF guarantee. Here, we consider the case that $K=3, M=N=2 d$, and $d>1$. It seems that the extension to the case of $K>3$ is difficult. However, the considered case is a practically important case that allows perfect interference alignment.

Theorem 1: For $K=3$ and $M=N=2 d$, with properly chosen $\left\{\mathbf{A}_{k l}\right\}$, the matrix $\tilde{\mathbf{H}}$ is almost surely rank-deficient with nullity of $d$ (i.e., there exists a $K N d \times d$ matrix $\breve{\mathbf{V}}$ with full column rank such that $\tilde{\mathbf{H}} \breve{\mathbf{V}}=\mathbf{0}$ ) for randomly realized $\left\{\mathbf{H}_{k l}\right\}$. Further, let $\breve{\mathbf{V}}=\left[\breve{\mathbf{V}}_{1}^{T}, \breve{\mathbf{V}}_{2}^{T}, \breve{\mathbf{V}}_{3}^{T}\right]^{T}$ be the partition of $\breve{\mathbf{V}}$ with $N d \times d$ submatrices $\breve{\mathbf{V}}_{l}, l \in\{1,2,3\}$. Then, we have

$$
\breve{\mathbf{V}}_{l}=\left[\mathbf{a}_{1} \otimes \mathbf{g}_{1}, \mathbf{a}_{2} \otimes \mathbf{g}_{2}, \ldots, \mathbf{a}_{d} \otimes \mathbf{g}_{d}\right]
$$

where $\mathbf{a}_{m}$ and $\mathbf{g}_{m}, m \in\{1, \ldots, d\}$, are $d \times 1$, and $N \times 1$ vectors, respectively, and $\left\{\mathbf{g}_{m}\right\}$ are linearly independent of each other.

Proof: See Appendix.

For $d>1$, the iterative algorithm in [9] selects one of the columns of (13) as the beamforming vectors for transmitter $l$, sectorizes the vector into $d$ subvectors of size $N$, and uses each subvector for each stream of the same transmitter. Suppose that $\mathbf{a}_{m} \otimes \mathbf{g}_{m}$ for a particular $m$ is chosen. Then

$$
\mathbf{a}_{m} \otimes \mathbf{g}_{m}=\left[\left[\mathbf{a}_{m}\right]_{1} \mathbf{g}_{m}^{T},\left[\mathbf{a}_{m}\right]_{2} \mathbf{g}_{m}^{T}, \ldots,\left[\mathbf{a}_{m}\right]_{d} \mathbf{g}_{m}^{T}\right]^{T}
$$

and $\left[\mathbf{a}_{m}\right]_{i} \mathbf{g}_{m}, i \in\{1, \ldots, d\}$, is the beamforming vector for the $i$ th stream in this case. Note that beamforming vectors in (14) are essentially identical. Each vector is a scaled version of the other, and this is the reason why the iterative algorithm in [9] does not guarantee the DoF and its performance is degraded when $d>1$. This problem can be overcome by choosing the beamforming vectors from different columns of $\breve{\mathbf{V}}_{l}$ in (13). For example, we can construct $\mathbf{V}_{l}$ for transmitter $l$ as follows:

$$
\mathbf{V}_{l}=\left[\left[\mathbf{a}_{1}\right]_{1} \mathbf{g}_{1},\left[\mathbf{a}_{2}\right]_{2} \mathbf{g}_{2}, \ldots,\left[\mathbf{a}_{d}\right]_{d} \mathbf{g}_{d}\right] .
$$

$$
\tilde{\mathbf{H}} \triangleq\left[\begin{array}{cccccc}
\mathbf{0} & \mathbf{I}_{\boldsymbol{d}} \otimes \mathbf{H}_{12} & -\mathbf{A}_{13} \otimes \mathbf{H}_{13} & \mathbf{0} & \cdots & \mathbf{0} \\
\mathbf{0} & \mathbf{I}_{d} \otimes \mathbf{H}_{12} & \mathbf{0} & -\mathbf{A}_{14} \otimes \mathbf{H}_{14} & \cdots & \mathbf{0} \\
\vdots & \vdots & \vdots & \vdots & \vdots & \vdots \\
\mathbf{0} & \mathbf{I}_{d} \otimes \mathbf{H}_{12} & \mathbf{0} & \cdots & \cdots & -\mathbf{A}_{1 K} \otimes \mathbf{H}_{1 K} \\
\mathbf{I}_{d} \otimes \mathbf{H}_{21} & \mathbf{0} & -\mathbf{A}_{23} \otimes \mathbf{H}_{23} & \mathbf{0} & \cdots & \mathbf{0} \\
\mathbf{I}_{d} \otimes \mathbf{H}_{21} & \mathbf{0} & \mathbf{0} & -\mathbf{A}_{24} \otimes \mathbf{H}_{24} & \cdots & \mathbf{0} \\
\vdots & \vdots & \vdots & \vdots & \vdots & \vdots \\
\mathbf{I}_{d} \otimes \mathbf{H}_{21} & \mathbf{0} & \mathbf{0} & \cdots & \cdots & -\mathbf{A}_{2 K} \otimes \mathbf{H}_{2 K} \\
\vdots & \vdots & \vdots & \vdots & \vdots & \vdots \\
\mathbf{I}_{d} \otimes \mathbf{H}_{K 1} & -\mathbf{A}_{K 2} \otimes \mathbf{H}_{K 2} & \mathbf{0} & \cdots & \cdots & \mathbf{0} \\
\vdots & \vdots & \vdots & \vdots & \vdots & \vdots \\
\mathbf{I}_{d} \otimes \mathbf{H}_{K 1} & \mathbf{0} & \cdots & \mathbf{0} & \cdots & \mathbf{0}
\end{array}\right]
$$


Now in (15), beamforming vectors for different streams are linearly independent and can be orthonormalized by the GramSchmidt procedure. The orthonormalized version of (15) can also serve as the beamforming vectors that enable perfect interference alignment by satisfying (6). This is because $\left\{\mathbf{A}_{k l}\right\}$ can be adjusted to incorporate the scaling associated with the orthonormalization. In summary, to enhance the iterative algorithm in [9], we here propose the construction of beamforming vectors as in (15) at each iteration and the orthonormalization of the beamforming vectors by the Gram-Schmidt procedure at the end of the iteration.

\section{PREDICTIVE BEAMFORMING FOR INTERFERENCE ALIGNMENT}

Now consider the time-varying channel case. In this case, the beamforming vectors for interference alignment over time-varying channels can be obtained by tracking the eigenvectors of $\tilde{\mathbf{H}}[n]^{H} \tilde{\mathbf{H}}[n]$. This is because the solution to (12) is given by the eigenvector of $\tilde{\mathbf{H}}[n]^{H} \tilde{\mathbf{H}}[n]$ corresponding to the smallest eigenvalue. The basis tracking for a signal subspace and the tracking of signal eigenvectors have been extensively investigated in the areas of temporal and spatial domain spectral analysis [26]-[30]. In these areas, efficient tracking algorithms have been derived by exploiting the structure of sample covariance matrices. However, the application of such techniques to tracking the beamforming vectors for interference alignment does not seem feasible because beamforming for interference alignment is not based on the sample covariance matrix but on channel estimates. In this section, we propose the use of the perturbation theory for eigenvectors [31] for tracking interference-aligning beam vectors. The idea is described as follows: given $\mathbf{R}[m] \triangleq \tilde{\mathbf{H}}[m]^{H} \tilde{\mathbf{H}}[m]$ and its eigenvalues and eigenvectors at time $m$, we can predict the eigenvectors at time $n$ for $n>m$ if the elements of $\mathbf{R}[n]=\tilde{\mathbf{H}}[n]^{H} \tilde{\mathbf{H}}[n]$ exhibit only small changes as compared with those of $\mathbf{R}[\mathrm{m}]$. This type of prediction for beamforming vectors to align interference is possible in slowly fading channels. Next, we present two theorems regarding the prediction of beamforming vectors for interference alignment. In the first theorem, $\mathbf{R}[m]$ is assumed to have full rank and the result is obtained by directly applying the linear perturbation theory in [31]. The case of rank deficient $\mathbf{R}[m]$ is considered in the second theorem, where a more recent result on perturbation analysis in [32] is used.

Theorem 2: Suppose that $\mathbf{R}[\mathrm{m}]$ has the full rank of $r_{F}=K(K-2) M d$. Let $\mathbf{v}[m]$ be the least squares solution to (12), i.e., the eigenvector of $\mathbf{R}[\mathrm{m}]$ corresponding to the smallest eigenvalue $\lambda_{1}$ at time $m$. Then, the solution to (12) at time $n(n>m)$ can be written as

$$
\begin{aligned}
\mathbf{v}[n]= & \mathbf{v}[m]-\sum_{i=2}^{r_{F}} \frac{\mathbf{q}_{i}^{H} \mathbf{G}_{m}[n] \mathbf{v}[m]}{\lambda_{i}-\lambda_{1}} \mathbf{q}_{i} \\
& +\left\|\mathbf{G}_{m}[n]\right\|_{2}^{2} \sum_{j=2}^{r_{F}} \frac{1}{\left(\lambda_{j}-\lambda_{1}\right)} \\
& \times\left(\sum_{i=2}^{r_{F}} a_{i} \mathbf{q}_{j}^{H} \overline{\mathbf{G}}_{m}[n] \mathbf{q}_{i}-\dot{\lambda}_{1}(0) a_{j}\right) \\
& \times \mathbf{q}_{j}+o\left(\left\|\mathbf{G}_{m}[n]\right\|_{2}^{2}\right)
\end{aligned}
$$

where

$$
\mathbf{G}_{m}[n] \triangleq \mathbf{R}[n]-\mathbf{R}[m]=\tilde{\mathbf{H}}[n]^{H} \tilde{\mathbf{H}}[n]-\tilde{\mathbf{H}}[m]^{H} \tilde{\mathbf{H}}[m]
$$

$\overline{\mathbf{G}}_{m}[n] \triangleq \frac{\mathbf{G}_{m}[n]}{\left\|\mathbf{G}_{m}[n]\right\|_{2}} ;\left\{\lambda_{i}\right\}$ are the ordered eigenvalues of $\mathbf{R}[m]=\tilde{\mathbf{H}}[m]^{H} \tilde{\mathbf{H}}[m]$, which are distinct $\left(0<\lambda_{1}<\right.$ $\left.\lambda_{2}<\cdots<\lambda_{r_{F}}\right)$, and $\left\{\mathbf{q}_{i}\right\}$ are the corresponding eigenvectors $\left(\mathbf{q}_{1}=\mathbf{v}[m]\right) ; a_{i}=\frac{\mathbf{q}_{i}^{H} \overline{\mathbf{G}}_{m}[n] \mathbf{v}[m]}{\boldsymbol{\lambda}_{i}-\lambda_{1}}$ and $\dot{\lambda}_{1}(0)=\mathbf{v}[m]^{H} \overline{\mathbf{G}}_{m}[n] \mathbf{v}[m]$.

Proof: See Appendix.

We use the first and second terms in the RHS of (16) for predicting $\mathbf{v}[n]$. Specifically, let $\overline{\mathbf{v}}[n]$ denote the prediction of $\mathbf{v}[n]$. Then,

$$
\overline{\mathbf{v}}[n]=\mathbf{v}[m]-\sum_{i=2}^{r_{F}} \frac{\mathbf{q}_{i}^{H} \mathbf{G}_{m}[n] \mathbf{v}[m]}{\lambda_{i}-\lambda_{1}} \mathbf{q}_{i}
$$

The third term in the RHS of (16) represents the dominant prediction error and is denoted as $E_{F R}(m, n)$.

Corollary 1: The dominant prediction error in (16), $E_{F R}(m, n)$, is upper bounded as

$$
\begin{aligned}
& \left\|E_{F R}(m, n)\right\|^{2} \leq\left\|\mathbf{G}_{m}[n]\right\|_{2}^{4} \\
& \times\left\{\left(\sum_{j=2}^{r_{F}} \frac{1}{\left(\lambda_{j}-\lambda_{1}\right)^{2}}\right)^{2}+\sum_{j=2}^{r_{F}} \frac{1}{\left(\lambda_{j}-\lambda_{1}\right)^{4}}\right\} .
\end{aligned}
$$

Proof: $\left\|E_{F R}(m, n)\right\|$ is the norm of the third term in the RHS of (16). By using the subadditivity and submultiplicity

$$
\begin{aligned}
&\left\|E_{F R}(m, n)\right\|^{2} \leq\left\|\mathbf{G}_{m}[n]\right\|_{2}^{4} \sum_{j=2}^{r_{F}} \frac{1}{\left(\lambda_{j}-\lambda_{1}\right)^{2}} \\
& \quad \times\left|\sum_{i=2}^{r_{F}} a_{i} \mathbf{q}_{j}^{H} \overline{\mathbf{G}}_{m}[n] \mathbf{q}_{i}-\dot{\lambda}_{1}(0) a_{j}\right|^{2}\left\|\mathbf{q}_{j}\right\|^{2} \\
& \leq\left.\left\|\mathbf{G}_{m}[n]\right\|\right|_{2} ^{4} \sum_{j=2}^{r_{F}} \frac{1}{\left(\lambda_{j}-\lambda_{1}\right)^{2}} \\
& \quad \times\left(\sum_{i=2}^{r_{F}}\left|a_{i}\right|^{2}\left|\mathbf{q}_{j}^{H} \overline{\mathbf{G}}_{m}[n] \mathbf{q}_{i}\right|^{2}+\left|\dot{\lambda}_{1}(0)\right|^{2}\left|a_{j}\right|^{2}\right) \\
& \leq\left\|\mathbf{G}_{m}[n] \mid\right\|_{2}^{4} \sum_{j=2}^{r_{F}} \frac{1}{\left(\lambda_{j}-\lambda_{1}\right)^{2}} \\
& \times\left(\sum_{i=2}^{r_{F}}\left|a_{i}\right|^{2}+\left|a_{j}\right|^{2}\right) .
\end{aligned}
$$

Here, the last inequality holds because $\left|\mathbf{q}_{j} \overline{\mathbf{G}}_{m}[n] \mathbf{q}_{i}\right|^{2} \leq$ $\left\|\mathbf{q}_{j}\right\|^{2}\left\|\overline{\mathbf{G}}_{m}[n]\right\|_{2}^{2}\left\|\mathbf{q}_{i}\right\|^{2}=1$ and $\left(\dot{\lambda_{1}}(0)\right)^{2} \leq$ $\|\mathbf{v}[m]\|^{2}\left\|\overline{\mathbf{G}}_{m}[n]\right\|_{2}^{2}\|\mathbf{v}[m]\|^{2}=1$. The RHS of (20) is less than or equal to the RHS of (19) because $\left|a_{i}\right|^{2} \leq \frac{\left\|\mathbf{q}_{i}\right\|^{2}\left\|\overline{\mathbf{G}}_{m}[n]\right\|_{2}^{2}\|\mathbf{v}[m]\|^{2}}{\left(\lambda_{1}-\lambda_{i}\right)^{2}}=\frac{1}{\left(\lambda_{1}-\lambda_{i}\right)^{2}}$.

The upper bound in (19) is given by a function of $\left\|\mathbf{G}_{m}[n]\right\|_{2}^{2}$ and $\left\{\frac{1}{\left(\lambda_{j}-\lambda_{1}\right)^{2}} \mid j=2, \ldots, r_{F}\right\}$.

Theorem 3: Suppose that $\mathbf{R}[m]$ is rank-deficient with rank $r$. Let $\mathbf{V}[m]$ be a matrix whose columns form the null space 
of $\mathbf{R}[m]$. Then, the perturbed null space $\mathbf{V}[n]$ for $\mathbf{R}[n]$ at time $n(>m)$ is given by

$$
\mathbf{V}[n]=\mathbf{V}[m]-\sum_{i=1}^{r} \frac{\mathbf{q}_{i} \mathbf{q}_{i}^{H} \mathbf{G}_{m}[n] \mathbf{V}[m]}{\lambda_{i}}+O\left(\left\|\mathbf{G}_{m}[n]\right\|_{2}^{2}\right) .
$$

For an arbitrary null vector $\mathbf{v}[n]$ of $\mathbf{R}[n]$, we have

$$
\begin{aligned}
\mathbf{v}[n]= & \mathbf{v}[m]-\sum_{i=1}^{r} \frac{\mathbf{q}_{i}^{H} \mathbf{G}_{m}[n] \mathbf{v}[m]}{\lambda_{i}} \mathbf{q}_{i} \\
& +\left\|\mathbf{G}_{m}[n]\right\|_{2}^{2} \sum_{j=1}^{r} \frac{1}{\lambda_{j}} \\
& \times\left(\sum_{i=1}^{r} a_{i} \mathbf{q}_{j}^{H} \overline{\mathbf{G}}_{m}[n] \mathbf{q}_{i}\right) \mathbf{q}_{j} \\
& +o\left(\left\|\mathbf{G}_{m}[n]\right\|_{2}^{2}\right)
\end{aligned}
$$

where $\mathbf{G}_{m}[n]$ is defined in (17); $a_{i}=\frac{\mathbf{q}_{i}^{H} \overline{\mathbf{G}}_{m}[n] \mathbf{v}[m]}{\lambda_{i}} ;\left\{\lambda_{i}\right\}$ are ordered eigenvalues of $\mathbf{R}[m]$ that are not necessarily distinct $\left(0<\lambda_{1} \leq \lambda_{2} \leq \cdots \leq \lambda_{r}\right)$, and $\left\{\mathbf{q}_{i}\right\}$ are the corresponding eigenvectors.

Proof: See Appendix.

Again the first and second terms of RHS of (22) are used for prediction, and the third term represents the dominant prediction error. The prediction of $\mathbf{v}[n]$ is given by

$$
\overline{\mathbf{v}}[n]=\mathbf{v}[m]-\sum_{i=1}^{r} \frac{\mathbf{q}_{i}^{H} \mathbf{G}_{m}[n] \mathbf{v}[m]}{\lambda_{i}} \mathbf{q}_{i} .
$$

Corollary 2: The dominant prediction error in (22), denoted by $E_{R D}(m, n)$, is upper bounded as

$$
\left\|E_{R D}(m, n)\right\|^{2} \leq\left\|\mathbf{G}_{m}[n]\right\|_{2}^{4}\left(\sum_{j=1}^{r} \frac{1}{\lambda_{j}^{2}}\right)^{2} .
$$

This inequality can be proved in a manner similar to the case of Corollary 1. In (19) and (24), the impact of $\mathbf{G}_{m}[n]$ and $\mathbf{R}[m]$ on prediction error is separated. As expected, the MSE of beam prediction increases as $n$ progresses, and the increase rate is given by $\left\|\mathbf{G}_{m}[n]\right\|_{2}^{4}$ in both cases. Interestingly, the impact of $\mathbf{R}[m]$ at the reference time is seen by the spectral gap of $\mathbf{R}[m]$, i.e., the difference between the two distinct smallest eigenvalues; when the spectral gap is large, the tracking performance is good.

The perturbation form (22) of the null vector in Theorem 3 is very similar to the result of Theorem 2 . Note that the result obtained by substituting $0=\lambda_{1}<\lambda_{2}<\cdots<\lambda_{r_{F}}$ into (16) is identical to (22) in the rank-deficient case with a nullity of one, i.e., $r_{F}=r+1$. In this case, the zero eigenvalue has a multiplicity of one and, thus, Theorem 2 holds. When the nullity is larger than one, as in the example of $M=N=4$, $K=3$ or $M=2, N=3$, and $K=3$, however, the assumption for the derivation of Theorem 2 [12] is broken, and the general result of Theorem 3 should be applied. Thus, the newly derived update formula in Theorem 3 is a generalized version for the null vector update in a rank-deficient case with arbitrary nullity. Theorems 2 and 3 provide a basis for a predictive beam solution to interference alignment in slowly fading channels. Once the full eigen-decomposition of $\mathbf{R}[m]$ is performed at time $m$, the beam solutions at successive time steps $n=m+1, m+2, \ldots, m+L-1$ are obtained by these formulas where $L$ is the prediction depth. In the proposed algorithm, the full eigen-decomposition is performed once in every $L$ time steps and linear prediction is performed in between two full eigen-decompositions. During the prediction period, we only need to compute the channel difference matrix and perform matrix additions and multiplications. The value of prediction depth $L$ depends on the fading rate (or mobile speed) and allowable prediction error. In typical slow fading, the value of $L$ can be large (which will be seen in Section V), and the reduction of computational complexity can be significant as compared with the method performing full eigen-decomposition at each time. The proposed algorithm is summarized below.

1) The Proposed Algorithm: At $n=i L$ for some integer $L$ and $i=0,1, \ldots$ (eigen-decomposition phase)

$1 \quad$ Initialize $\mathbf{A}_{k l}^{(i)}=\mathbf{I}$ for $i=0$ and $\mathbf{A}_{k l}^{(i)}=\mathbf{A}_{k l}^{(i-1)}$ for $i>0$.

2 Construct $\tilde{\mathbf{H}}[i L]$ with $\left\{\mathbf{A}_{k l}^{(i)}\right\}$ and available $\left\{\mathbf{H}_{k l}[i L]\right\}$; update $\left\{\mathbf{A}_{k l}^{(i)}\right\}$ by solving (11) and (12) iteratively until it converges. (A proper normalization step can be added here.)

3 Reconstruct $\tilde{\mathbf{H}}[i L]$ with $\left\{\mathbf{A}_{k l}^{(i)}\right\}$ obtained in Step 2.

4 Perform the eigen-decomposition of $\mathbf{R}[i L]=$ $\tilde{\mathbf{H}}[i L]^{H} \tilde{\mathbf{H}}[i L]$.

5 Set $\mathbf{v}[i L]$ to the eigenvector associated with the smallest eigenvalue.

for $n=i L+1: i L+L-1$ (prediction phase)

6 Construct $\tilde{\mathbf{H}}[n]$ using $\left\{\mathbf{A}_{k l}^{(i)}\right\}$ obtained in Step 2 and $\left\{\mathbf{H}_{k l}[n]\right\}$.

7 Compute $\mathbf{G}_{i L}[n]=\tilde{\mathbf{H}}[n]^{H} \tilde{\mathbf{H}}[n]-\tilde{\mathbf{H}}[i L]^{H} \tilde{\mathbf{H}}[i L]$, and update the beam vectors using either (18) or (23) to obtain $\overline{\mathbf{v}}[n]$ depending on the rank of $\mathbf{R}[i L]$. (The rank is obtained in Step 4.)

end

Since $\mathbf{A}_{k l}^{(i)}=\mathbf{A}_{k l}^{(i-1)}$ for $i>0$ in Step 1, updating $\left\{\mathbf{A}_{k l}^{(i)}\right\}$ in Step 2 requires only a few iterations to converge. Some additional remarks of interest are as follows:

- As mentioned in Section II-B, an orthonormalization step can be added to equalize power among the streams and users without disturbing the interference subspace. During the prediction phase, an orthonormalization step can be inserted after obtaining $\overline{\mathbf{v}}[n]$ in Step 7.

- We can consider one modification of the proposed algorithm to incorporate signal power as well as the minimization of interference, which was considered in [9]. In this modification, $\mathbf{R}^{\prime}[n] \triangleq \tilde{\mathbf{H}}[n]_{\tilde{\mathbf{H}}}^{H} \tilde{\mathbf{H}}[n]-\gamma \boldsymbol{\Phi}[n]^{H} \boldsymbol{\Phi}[n]$ is used instead of $\mathbf{R}[n]=\tilde{\mathbf{H}}[n]^{H} \tilde{\mathbf{H}}[n]$, where $\gamma$ is a weighting factor and $\boldsymbol{\Phi}[n] \triangleq \operatorname{diag}\left(\mathbf{I}_{d} \otimes \mathbf{H}_{11}[n], \ldots, \mathbf{I}_{d} \otimes \mathbf{H}_{K K}[n]\right)$. In this case, $\mathbf{R}^{\prime}[n]$ almost surely has full rank, and Theorem 2 needs to be applied.

- In the proposed algorithm, we assume that the variation of $\left\{\mathbf{A}_{k l}^{(i)}\right\}$ is small enough to be negligible. Without this assumption, a step to update $\left\{\mathbf{A}_{k l}^{(i)}\right\}$ with (11) should be included after Step. 7. However, we do not update $\left\{\mathbf{A}_{k l}^{(i)}\right\}$ in the prediction phase because exclusion of this step does 


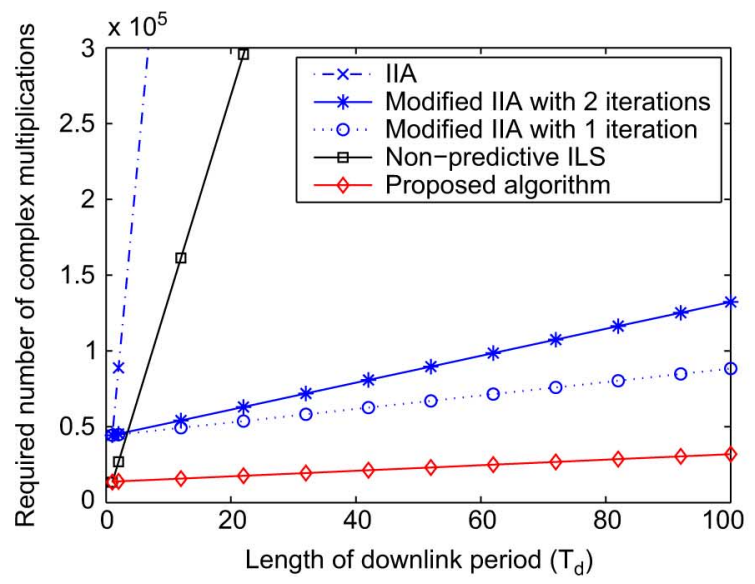

(a)

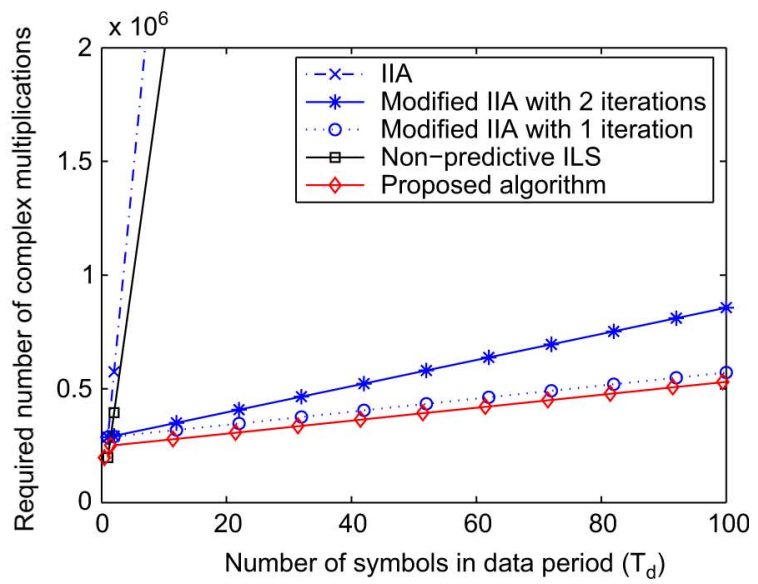

(b)

Fig. 1. Complexity comparison (a) $M=N=2, K=3, d=1$. (b) $M=N=4, K=3, d=2$.

not cause performance loss much in terms of the sum throughput.

- In the feasible multiple-stream case $(d>1)$, the $d$ eigenvectors associated with the $d$ smallest eigenvalues of $\mathbf{R}[i L]$ are obtained in the eigen-decomposition phase, and each of the eigenvectors is updated according to (23) in the prediction phase. Then, a beamforming matrix with linearly independent $d$ columns can be obtained with these $d$ eigenvectors, as explained in Section II-B.

\section{Performance AnAlysis AND ApPlication}

In this section, we examine the overall performance by considering both the beam prediction and channel prediction with an application to TDD downlink. To implement the proposed predictive beam design, channel state information is required at the transmitters, and channel prediction should be performed in the case of TDD operation. Therefore, the prediction error results from two sources: one is the predictive beam computation itself and the other is imperfect channel knowledge due to the use of a channel predictor or estimator to obtain the channel information.

\section{A. Channel Model, Frame Structure, and Application}

To investigate the impact of channel dynamics and estimation on the prediction performance, we need a time-varying channel model. In this paper, we use the state-space channel model with zero-mean Gaussian channel gain, i.e., the firstorder Gauss-Markov channel model, which has widely been used as a good model for Rayleigh-fading time-varying channels [33]-[35]. The channel gain between antenna $j$ of transmitter $l$ and antenna $i$ of receiver $k$, i.e., the $(i, j)$ th element of $\mathbf{H}_{k l}[n]$, is given by

$$
H_{k l}^{i j}[n]=\beta_{k l} H_{k l}^{i j}[n-1]+u_{k l}^{i j}[n], n \geq 1
$$

where $H_{k l}^{i j}[0] \sim \mathcal{C N}\left(0, \Pi_{0}^{2}\right), \beta_{k l} \in(0,1]$ is the correlation coefficient, and $u_{k l}^{i j}[n] \sim \mathcal{C} \mathcal{N}\left(0, \Pi_{0}^{2}\left(1-\beta_{k l}^{2}\right)\right)$ is the plant noise of the channel process. The parameter $\beta_{k l}$ depends on the Doppler spread and symbol duration [34], [35]. Note that $\beta_{k l}$ depends on indices $k$ and $l$ of transmitter and receiver but not on antenna indices $i$ and $j$ of the same transmitter-receiver pair since the fading rate is determined by the relative speed between the transmitter and receiver. For simplicity, we here assume that each element in the MIMO link evolves independently and the statistics of channel process are identical for all $i, j$ and $k, l$.

We assume that the proposed interference alignment is applied to TDD downlink transmission with one base station controller (BSC) and $K$ pairs of base station (BS) and mobile station (MS). It is assumed that the TDD frame size is $L^{\prime}(=$ $\left.T_{u}+T_{d}\right)$, and $T_{u}$ and $T_{d}$ are the numbers of uplink and downlink symbols, respectively. To compute interference-aligning beam vectors at the BSC, $K(K-1)$ channel matrices corresponding to all MIMO links between $K$ BSs and $K$ MSs are required ${ }^{2}$. To obtain CSI at BSs, pilot symbols can be embedded in the uplink, and the length $T_{u}$ of uplink transmission can be set to be long enough to guarantee that the pilot sequence of one uplink antenna is orthogonal to those from all others. Then, the obtained channel information can be sent to the BSC for the computation of interference-aligning beam vectors. Under this scenario, the channel can be estimated at the transmitter directly due to channel reciprocity. For the period of uplink pilot symbols, the received signal at antenna $j$ of transmitter $l$ is given by

$$
\vec{y}_{l}^{j}[n]=H_{k l}^{i j}[n] p_{k}^{i}[n]+\vec{w}_{l}^{j}[n], n \in \text { uplink }
$$

where $p_{k}^{i}[n]$ denotes the known pilot symbol from antenna $i$ of receiver $k$ in the uplink, $\vec{H}_{l k}^{j i}=H_{k l}^{i j}[n]$ due to the channel reciprocity, and $\vec{w}_{l}^{j}[n] \sim \mathcal{C N}\left(0, \sigma^{2}\right)$ is the additive white Gaussian noise at time $n$. Equations (25) and (26) form a state-space channel model to which Kalman filtering can be applied to obtain an optimal channel estimate. The optimal estimation under the state-space model was thoroughly studied by Dong et al. [33]. The optimal estimation is given by Kalman filtering during the uplink pilot period and Kalman prediction during the downlink data phase

$$
\hat{H}_{k l}^{i j}[m+p]=\beta_{k l}^{p} \hat{H}_{k l}^{i j}[m], p=1, \ldots, T_{d}
$$

${ }^{2}$ For interference alignment, $K$ desired links from each transmitter to a corresponding receiver are not required. To incorporate signal power in the beam design, the desired links are also required. 
where $\hat{H}_{k l}^{i j}[m]$ is the channel estimate at time $m$. Closed-form expressions for the steady-state channel estimation MSE of pilot and data phases are known and available in [33].

\section{B. Sum Rate Loss}

In Section III, the beam prediction performance is analyzed and the dominant error term is obtained under the assumption of perfect channel knowledge. In practical systems like the TDD transmission explained in the previous subsection, the MSE of beam prediction should further include the channel estimation error. The expected MSE of beam prediction averaged over channel realizations can be used to assess the behavior of beam design MSE and corresponding sum rate loss as functions of fading rate and prediction depth. Both $\mathbb{E}\left\|E_{F R}(m, n)\right\|^{2}$ and $\mathbb{E}\left\|E_{R D}(m, n)\right\|^{2}$ in (19) and (24) are composed of two components: one depends on the eigenvalues of $\mathbf{R}[m]$ at reference time $m$ and the other relies on the channel difference matrix $\mathbf{G}_{m}[n]$ between two time steps, $m$ and $n$. The expected eigenvalues of $\tilde{\mathbf{H}}[m]$ and, thus, those of $\mathbf{R}[m]$ in (19) and (24) depend only on time $m$, and they are dominantly affected by the size of $\tilde{\mathbf{H}}$, i.e., the numbers of users, transmit streams, and antennas. Under the considered channel model in Section IV-A, $\mathbb{E}\left\|\hat{\mathbf{G}}_{m}[m+p]\right\|_{2}$, $p=1, \ldots, T_{d}$, where $\hat{\mathbf{G}}_{m}[n] \triangleq \tilde{\hat{\mathbf{H}}}[n]^{H} \tilde{\hat{\mathbf{H}}}[n]-\tilde{\hat{\mathbf{H}}}[m]^{H} \tilde{\hat{\mathbf{H}}}[m]$ and $\hat{\mathbf{H}}[n]$ is in the form of (7) with estimated channels $\left\{\hat{\mathbf{H}}_{i j}\right\}$ instead of true channels $\left\{\mathbf{H}_{i j}\right\}$, can be obtained explicitly, and is given in the following theorem.

Theorem 4: Under the assumption of the Gauss-Markov channel model and $\beta_{k l}=\beta$ for all $k$ and $l$, the upper bound of the expected 2-norm of $\hat{\mathbf{G}}_{m}[m+p]$ is given by

$$
\mathbb{E} \mid \hat{\mathbf{G}}_{m}[m+p] \|_{2} \leq\left(1-\beta^{2 p}\right)\left(1+\sqrt{6 \sigma_{a}^{4}} d\right) \Pi_{0}^{2} K(K-2) M N d
$$

where $\sigma_{a}^{4}$ is the maximum of the fourth moment of $\left[\mathbf{A}_{k l}\right]_{i j}$ viewed as a function of channel random variables under the assumption of its existence.

Proof: See Appendix.
Note that the overall beam design MSE in this case can be upperbounded using (28). It is seen from Theorem 4 that the tracking error decreases with the fading coefficient $\beta$ and increases with other parameters including $p, \Pi_{0}, K$, etc. In particular, with fixed $M, N, K, d$, and $\Pi_{0}$, the MSE of tracking error during the prediction period is given by $c\left(1-\beta^{2 p}\right)$ with some constant $c$; that is, the tracking error does not increase without bound, but saturates as time elapses.

Now we analyze the sum rate loss caused by both predictive beam design and channel estimation under the interference alignment condition in the case of a single data stream. (Similar analysis for multiple streams for one user is possible, but it does not provide more insight.) We assume that a zero-forcing (ZF) receive beam vector $\hat{\mathbf{u}}_{k}$ obtained based on the predicted channel is used at the receiver, and the zero-forced received signal is given by (29) at the bottom of the page, where $\check{\mathbf{H}}_{k l}\left(\triangleq \mathbf{H}_{k l}-\right.$ $\left.\hat{\mathbf{H}}_{k l}\right)$ is the channel prediction (or estimation) error, $\check{\mathbf{v}}_{k}\left(\triangleq \overline{\hat{\mathbf{v}}}_{k}-\right.$ $\hat{\mathbf{v}}_{k}$ ) denotes the error between beam $\hat{\mathbf{v}}_{k}$ correctly matched to the predicted channel and predictively obtained beam $\overline{\hat{\mathbf{v}}}_{k}$ using the predicted channel $\left\{\hat{\mathbf{H}}_{k l}\right\}$, and $n_{k}^{\prime}=\hat{\mathbf{u}}_{k}^{H} \mathbf{n}_{k}\left(\sim \mathcal{C N}\left(0, \sigma^{2}\right)\right)$. In the last equality, we use the fact that $\hat{\mathbf{u}}_{k} \hat{\mathbf{H}}_{k l} \hat{\mathbf{v}}_{l}=0$ by interference alignment for the exact beam based on the predicted channel. In (29), the first term stands for the desired signal component, the second and third terms are the interference from channel and beam vector predictions, respectively. The loss of the expected sum rate can then be defined as (30) at the bottom of the page, where $I_{h, k}=\sum_{l=1, l \neq k}^{K}\left|\hat{\mathbf{u}}_{k}^{H} \check{\mathbf{H}}_{k l} \hat{\mathbf{v}}_{l}\right|^{2}$ and $I_{v, k}=\sum_{l=1}^{K}\left|\hat{\mathbf{u}}_{k}^{H} \mathbf{H}_{k l} \check{\mathbf{v}}_{l}\right|^{2}$. An upper bound of the expected rate loss due to channel prediction (or estimation) and beam prediction is obtained and given in the following theorem.

Theorem 5: Assuming independent and identically distributed (i.i.d.) channel coefficients with zero mean and variance $\Pi_{0}^{2}$ for all elements of all MIMO channel matrices, we have an upper bound of the expected sum rate loss, given by

$$
\Lambda \leq K \log \left(1+\frac{(K-1) M N \sigma_{h}^{2}+K M N \Pi_{0}^{2} \sigma_{v}^{2}}{\sigma^{2}}\right)
$$

$$
\begin{aligned}
\hat{\mathbf{u}}_{k}^{H} \mathbf{y}_{k} & =\hat{\mathbf{u}}_{k}^{H} \mathbf{H}_{k k}\left(\hat{\mathbf{v}}_{k}+\check{\mathbf{v}}_{k}\right) s_{k}+\sum_{l=1, l \neq k}^{k} \hat{\mathbf{u}}_{k}^{H} \mathbf{H}_{k l}\left(\hat{\mathbf{v}}_{l}+\check{\mathbf{v}}_{l}\right) s_{l}+\hat{\mathbf{u}}_{k}^{H} \mathbf{n}_{k} \\
& =\hat{\mathbf{u}}_{k}^{H} \mathbf{H}_{k k}\left(\hat{\mathbf{v}}_{k}+\check{\mathbf{v}}_{k}\right) s_{k}+\sum_{l=1, l \neq k}^{K} \hat{\mathbf{u}}_{k}^{H}\left(\hat{\mathbf{H}}_{k l}+\check{\mathbf{H}}_{k l}\right) \hat{\mathbf{v}}_{l} s_{l}+\sum_{l=1, l \neq k}^{K} \hat{\mathbf{u}}_{k}^{H} \mathbf{H}_{k l} \check{\mathbf{v}}_{l} s_{l}+n_{k}^{\prime} \\
& =\hat{\mathbf{u}}_{k}^{H} \mathbf{H}_{k k} \hat{\mathbf{v}} s_{k}+\sum_{l=1, l \neq k}^{K} \hat{\mathbf{u}}_{k}^{H} \check{\mathbf{H}}_{k l} \hat{\mathbf{v}}_{l} s_{l}+\sum_{l=1}^{K} \hat{\mathbf{u}}_{k}^{H} \mathbf{H}_{k l} \check{\mathbf{v}}_{l} s_{l}+n_{k}^{\prime}
\end{aligned}
$$

$$
\Lambda \triangleq \mathbb{E} \sum_{k=1}^{K} \log \left(1+\frac{\left|\mathbf{u}_{k}^{H} \mathbf{H}_{k k} \mathbf{v}_{k}\right|^{2}}{\sigma^{2}}\right)-\mathbb{E} \sum_{k=1}^{K} \log \left(1+\frac{\left|\hat{\mathbf{u}}_{k}^{H} \mathbf{H}_{k k} \hat{\mathbf{v}}_{k}\right|^{2}}{I_{h, k}+I_{v, k}+\sigma^{2}}\right)
$$




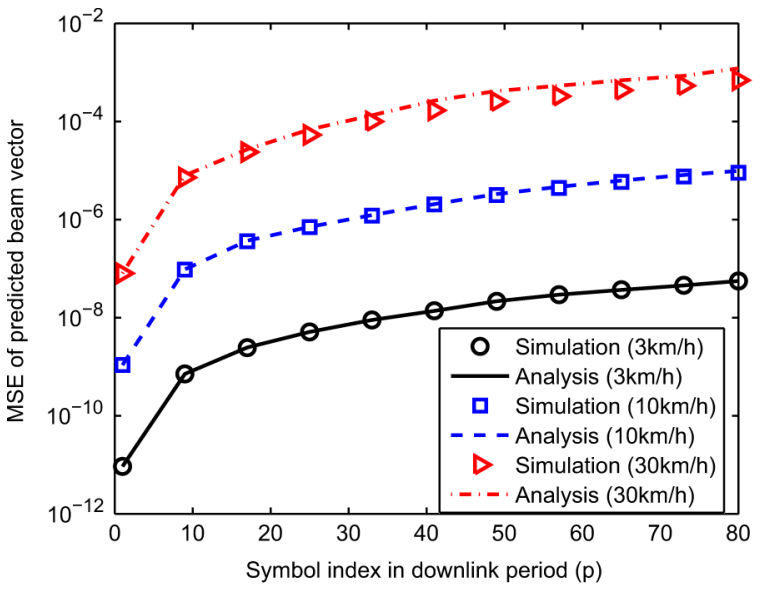

(a)

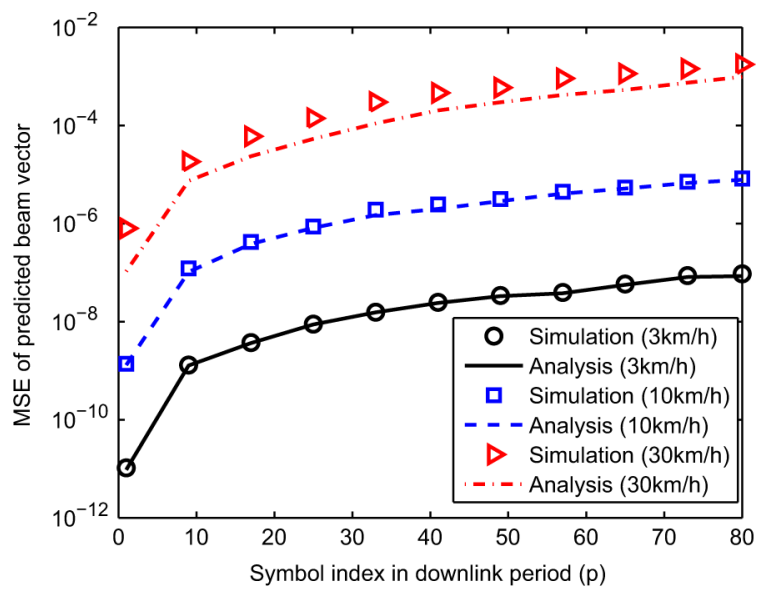

(b)

Fig. 2. MSE of beam vector prediction under different mobile speeds (a) $M=2, N=3, K=3, d=1$. (b) $M=N=2, K=3, d=1$.

TABLE I

Complexity of the Proposed Algorithm $(M=N=2 d, K=3)$

\begin{tabular}{|l|c|}
\hline Step & Complex multiplications \\
\hline 2- Compute $\tilde{\mathbf{H}}^{H} \tilde{\mathbf{H}}$ & $\left(J_{2}-1\right)\left\{3 N^{2}(N+1)+3\left(d^{2}+1\right) N^{3}+\frac{3}{2} d^{2}(d+1)\right\}$ \\
\hline 2- Iterative QR method & $\left(J_{2}-1\right)\left\{L_{Q R}\left(3 N d(3 N d-d+1)+\frac{2 d^{3}-3 d^{2}+d}{6}\right)\right\}$ \\
\hline 2- Determine $\left\{\mathbf{A}_{k l}^{(i)}\right\}$ & $\left(J_{2}-1\right)\left\{3\left(2 N^{2} d+3 N d^{2}+d^{3}\right)\right\}$ \\
\hline 3, 4 - Compute $\mathbf{H}^{H} \tilde{\mathbf{H}}$ & $3 N^{2}(N+1)+3\left(d^{2}+1\right) N^{3}+\frac{3}{2} d^{2}(d+1)$ \\
\hline 4 - Eigenvalue decomposition & $\frac{13}{3}(K N d)^{3}$ \\
\hline 6 - Construct $\mathbf{G}_{m}[n]$ & $3 N^{2}(N+1)+3\left(d^{2}+1\right) N^{3}+\frac{3}{2} d^{2}(d+1)$ \\
\hline 7 - Update & $6(3 N d-d) N d+9(N d)^{2}$ \\
\hline
\end{tabular}

where $\sigma_{h}^{2}$ and $\sigma_{v}^{2}$ are the MSEs of channel estimation and beam prediction, respectively.

Proof: See Appendix.

Theorem 5 is general in the sense that the Gauss-Markov channel assumption is not required, and it is valid for general channel estimator and beam design. In the case of the Gauss-Markov channel model, the channel estimation MSE $\sigma_{h}^{2}$ is known in [33], and an upperbound of beam prediction MSE $\sigma_{v}^{2}$ is given by (19), (24) and Theorem 4 . As expected, there is no loss if the channel estimation and beam tracking is perfect, i.e., $\sigma_{h}^{2}=\sigma_{v}^{2}=0$. It is interesting to see that the impact by beam design error $\sigma_{v}^{2}$ is scaled by the channel variance $\Pi_{0}^{2}$. The dominance of either term depends on the number of users and the variance of channel process.

\section{Complexity}

Here, we examine the computational complexity of the proposed algorithm and compare it with simple redesigning methods applying existing algorithms at every time step. For the redesigning methods, we consider the iterative interference alignment (IIA) algorithm proposed in [8], which has less complexity than the MAX-SINR algorithm in [8] and which is optimal at high SNR [36], and the iterative LS (ILS) approach [9] described in Section II-A. Here, we also considered the modified IIA algorithm to fit in slowly fading channels by initializing the transmitter and receiver beam vectors by the value of the previous time to reduce the number of iterations to a minimum. In this way, the modified IIA requires only a few iterations to converge and to yield the same performance

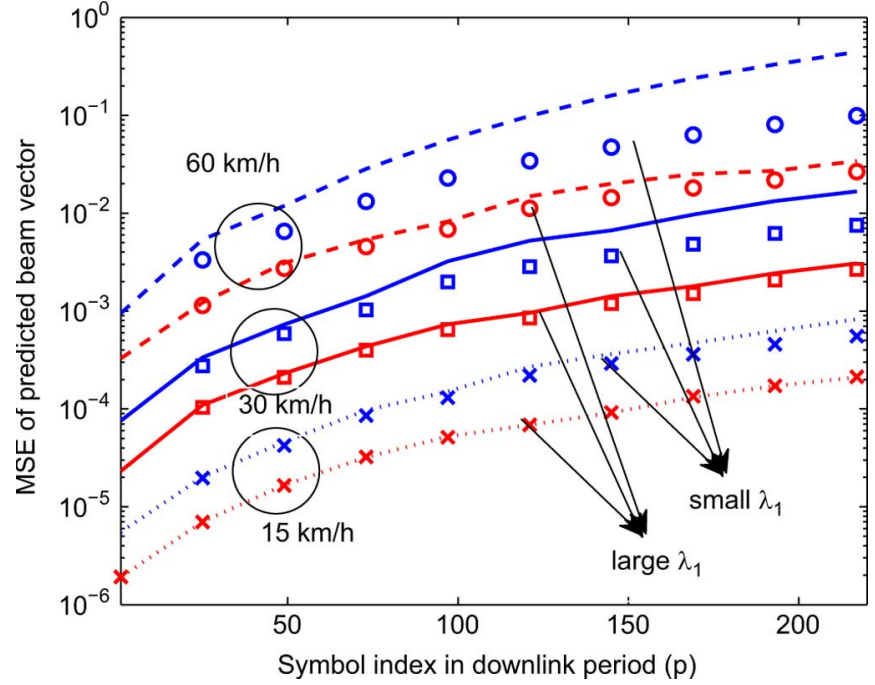

Fig. 3. MSE of beam vector prediction with two different channel realizations when $M=2, N=3, K=3$, and $d=1$. (marker: numerical MSE, line: analytical MSE).

as the proposed algorithm ${ }^{3}$. As a complexity measure, we use the number of required complex multiplications during one data period with $T_{d}$ symbols. We mainly consider the case of $N=M=2 d$ and $K=3$. The IIA algorithm requires $\mathcal{M}_{I I A}=$ $6\left(2 N^{2} d+2 N d\right)+6 L_{Q R}\left(N d(N-d+1)+\frac{2 d^{3}-3 d^{2}+d}{6}\right)$ in each iteration, where the first and second terms are the numbers of multiplications for computation of the interference covariance and $d$ extreme eigenvectors using the iterative QR method with $L_{Q R}$ iterations, respectively. Under the assumption that the numbers of iterations required to obtain a convergent solution with an arbitrary initialization and smart initialization are $J_{1}$ and two, respectively, the total numbers of multiplications of the redesigning and modified IIA for one data period are given by $T_{d} J_{1} \mathcal{M}_{I I A}$ and $\left(J_{1}+2\left(T_{d}-1\right)\right) \mathcal{M}_{I I A}$,

\footnotetext{
${ }^{3}$ For $M=N=2, d=1$, and $K=3$, the modified IIA with one or two iterations for beam update with this smart initialization has a sum rate loss of about 1 or $0.5 \frac{b p s}{H z}$, respectively, at $35 \mathrm{~dB}$ SNR under $3 \frac{\mathrm{km}}{\mathrm{h}}$ mobile speed. The proposed algorithm shows less than $0.5 \frac{b p s}{H z}$ rate loss under the same condition, as shown in Fig. 4(b), so we used two as the iteration number for the IIA method.
} 


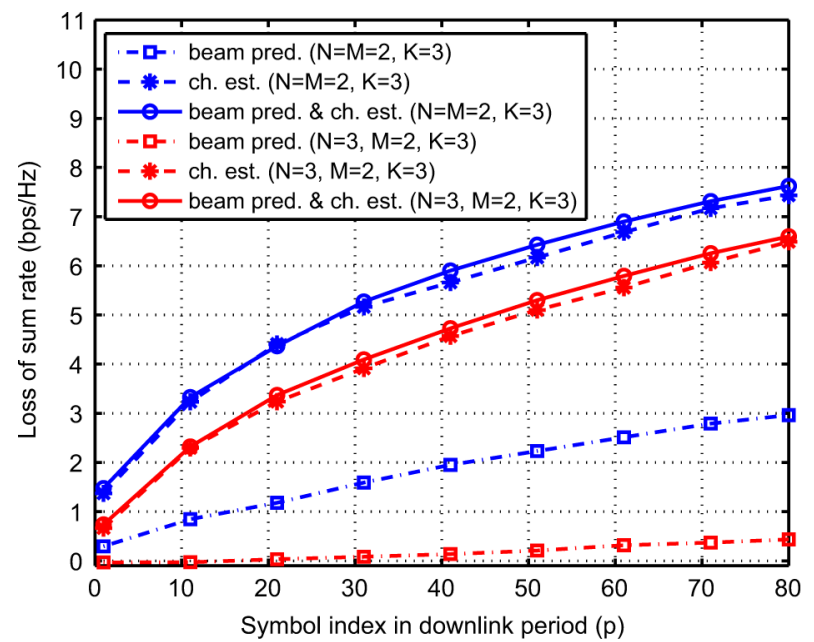

(a)

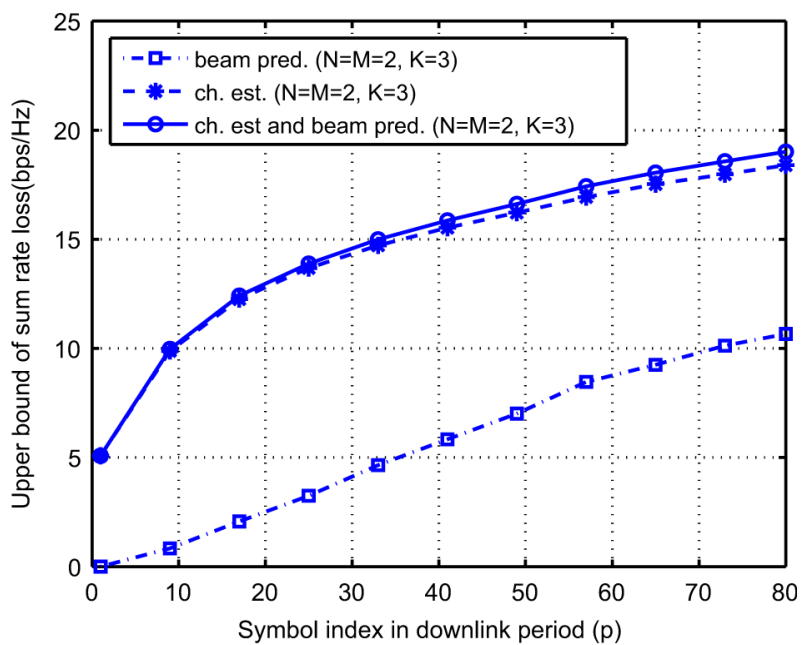

(b)

Fig. 4. (a) Sum rate losses with channel estimation and (or) beam prediction at different $p$ and $30 \frac{\mathrm{km}}{\mathrm{h}}$. (b) Upper bounds of sum rate loss obtained with (31) and analytical MSEs.

respectively. On the other hand, the redesigning ILS algorithm requires $\left(T_{d} J_{2}\right)\left\{3 N^{2}(N+1)+3\left(d^{2}+1\right) N^{3}+\frac{3}{2} d^{2}(d+\right.$ 1) $+L_{Q R}\left(3 N d(3 N d-d+1)+\frac{2 d^{3}-3 d^{2}+d}{6}\right)+3\left(2 N^{2} d+\right.$ $\left.\left.3 N d^{2}+d^{3}\right)\right\}$ operations, where $J_{2}$ is the number of iterations for ILS with an arbitrary initialization [9]. The first three terms are the complexity of obtaining $\tilde{\mathbf{H}}^{H} \tilde{\mathbf{H}}$, and the fourth and fifth terms are the complexity to obtain $d$ extreme eigenvectors using the iterative QR method and to update $\left\{\mathbf{A}_{k l}\right\}$, respectively. By exploiting the structure of $\tilde{\mathbf{H}}$, we further reduce the complexity of the ILS algorithm than the result in [9]. Now, consider the proposed predictive algorithm. The number of multiplications in each step is shown in Table I. Here, we assume that the full eigen-decomposition of $\mathbf{R}[m]$ is done by the Householder method to reduce the symmetric matrix to tri-diagonal form and the following QL algorithm to obtain the eigenvalues and eigenvectors. These two steps require $\frac{4}{3}(K N d)^{3}$ and $3(K N d)^{3}$ operations [37], and we simply assume that all these operations are complex multiplications. For $M=N=2, K=3, d=1$ and $M=N=4, K=3, d=2$, we plotted the complexity in Fig. 1. We used $L_{Q R}=15, J_{1}=100$, and $J_{2}=20$ to assure that both algorithms terminate with the same interference leakage of $10^{-4}$. As seen in Fig. 1, the proposed algorithm reduces the complexity significantly compared with redesigning approaches and shows less complexity than the best modified IIA method in slowly fading channels (and even the modified IIA method with only one iteration per step - the slope of the proposed algorithm is smaller). As $T_{d}$ increases, the complexity gain over the other methods increases linearly with respect to (w.r.t.) $T_{d}$ since the proposed method requires fewer operations in each update step than all the other methods. This is because iterative operations like the iterative QR method to obtain the extreme eigenvector(s) are required to update the beam vector in the other three algorithms. However, the proposed predictive beam design does not require any iterative operation during the update phase.

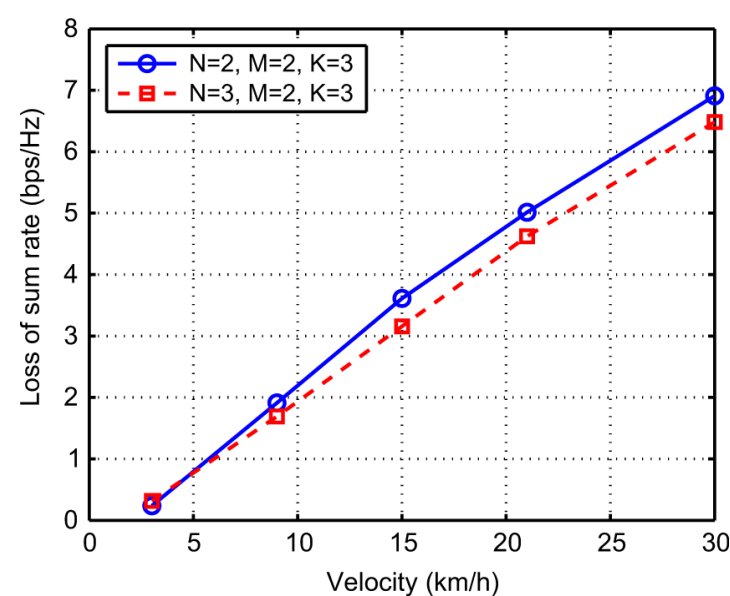

Fig. 5. Sum rate losses with channel estimation and beam prediction at different mobile speed and $p=T_{d}=80$.

\section{NuMERICAL Results}

To assess the performance of the proposed algorithm, we provide numerical results in this section. We assume $1.0 \mathrm{GHz}$ carrier frequency and a symbol duration of $66.7 \mu \mathrm{s}$, which is the length of one OFDM symbol in 3GPP long-term evolution (LTE). We used the channel and transmission model in Section IV-A with $L^{\prime}=100\left(T_{u}=20, T_{d}=80\right)$ and $\Pi_{0}^{2}=1$ for all simulations here. The fading coefficient $\beta$ of the GaussMarkov channel model is determined by the zero-order Bessel function of the first kind $\mathcal{J}_{0}\left(2 \pi f_{D} T\right)$, where $f_{D}$ and $T$ are the maximum Doppler frequency and symbol duration, respectively.

First, we validate the error analysis of the proposed algorithm based on the perturbations approach in Fig. 3 to Fig. 5. Since the solution to interference alignment is not unique, the interference misalignment $\|\tilde{\mathbf{H}} \mathbf{v}[n]\|^{2}$ is used as the measure of beam design MSE. When $M=2, N=3, K=3$, and $d=1$, the analytical MSEs match the numerical ones well for a wide range of mobile speed, as shown in Fig. 2(a), although the $\left\{\mathbf{A}_{k l}^{(i)}\right\}$ up- 
dating step is not included in the prediction phase of the proposed algorithm. In the case of $M=N=2, K=3$, and $d=1$, on the other hand, the $\left\{\mathbf{A}_{k l}^{(i)}\right\}$ updating step is included in the prediction phase of the proposed algorithm since interference alignment is feasible only with proper $\left\{\mathbf{A}_{k l}\right\}$ in this case. The numerical results again match the analysis well in this case, as shown in Fig. 2(b). Fig. 3 shows the MSE results according to mobile speed with two different channel realizations for $M=2, N=3, K=3$, and $d=1$ : one has a small minimum non-zero eigenvalue of $\tilde{\mathbf{H}}[\mathrm{m}]$, and the other has a large minimum nonzero eigenvalue of $\mathbf{H}[m]$. As expected by Corollaries 1 and 2, the beam prediction MSE is smaller when the minimum nonzero eigenvalue of $\tilde{\mathbf{H}}$ is larger, i.e., the spectral gap of $\tilde{\mathbf{H}}$ is larger. Fig. 4(a) shows the sum rate loss with the predictive beam design by the proposed algorithm and/or channel estimation by using the Kalman predictor at $35 \mathrm{~dB}$ SNR when $K=3, M=N=2, d=1$ and $K=3, M=2$, $N=3, d=1$. The rate loss increases with the symbol time since both $\sigma_{h}^{2}$ and $\sigma_{v}$ are increasing functions of $p$ in the form of $\left(1-\beta^{2 p}\right)$, as seen in Theorem 4 and [33]. So, it is seen that the increment of the rate loss monotonically decreases as $p$ increases. The rate loss for $M=2, N=3$, and $K=3$ is smaller than that for $M=N=2$ and $K=3$ since the former case has smaller MSE of beam prediction, as predicted by Theorem 5 . To verify Theorem 5, the upper bounds of sum rate loss with channel estimation and/or beam prediction MSEs are evaluated when $M=N=2$ and $K=3$. To obtain the upper bounds, we use (31). For the upper bound only with beam prediction error, we set $\sigma_{h}^{2}=0$. The upper bound only with channel estimation error is evaluated with $\sigma_{v}^{2}=0$. The upper bound of sum rate loss is not tight with the numerical result, but the behavior of each term is well predicted by Theorem 5; the relative contributions of predictive beam design and channel estimation errors to sum rate loss are similar in Fig. 4(a) and (b). Note also that the sum rate loss by channel estimation error is dominant compared with that by beam prediction. The rate loss w.r.t. different mobile speeds is also shown in Fig. 4. Under the slow-fading assumption $f_{D} T \ll 1$, the fading coefficient $\beta$ can be approximated as $\mathcal{J}_{0}\left(2 \pi f_{D} T\right)=\sum_{l=0}^{\infty} \frac{(-1)^{l}}{2^{2 l}(l !)^{2}}\left(2 \pi f_{D} T\right)^{2 l} \approx$ $1-\left(\pi f_{D} T\right)^{2}$. Then, $\left(1-\beta^{2 p}\right)$ can be approximated as $1-$ $\beta^{2 p} \approx 1-\left(1-\left(\pi f_{D} T\right)^{2}\right)^{2 p} \approx 2 p\left(\pi \frac{v_{m} T}{\chi}\right)^{2}$, where $v_{m}$ and $\chi$ are the mobile speed and the wavelength, respectively, since $(1-x)^{m}=1-m x+O\left(x^{2}\right)$. Thus, $\left(1-\beta^{2 p}\right)$ behaves like $v_{m}^{2}$, and $\Lambda$ behaves like $\log v_{m}^{2}$ at high SNR according to Theorems 4 and 5, as seen in Fig. 5.

Next, we compare the sum rate performance of the predictive beam design algorithm with that of others. We considered three different mobile speeds of 3,10 , and $30 \mathrm{~km} / \mathrm{h}$ and four different cases: two feasible cases with tall (or square) $\tilde{\mathbf{H}}[n](M=N=$ $2, K=3, d=1$ and $M=N=4, K=3, d=2$ ), a strictly fat $\tilde{\mathbf{H}}$ with one more transmit antenna, i.e., $M=2, N=3, K=3$, and $d=1$, and finally an infeasible case of $N=M=2$ and $K=4$. First, Fig. 6 shows the average sum rate w.r.t. average link SNR at $30 \mathrm{~km} / \mathrm{h}$ of six different approaches including the recalculating ILS redesigning transmit beams every time step, the proposed method, their modifications incorporating signal power where the weighting factor $\gamma$ is set by the inverse of SNR as in [9], the modified IIA, and the MAX-SINR algorithm. To see the relative performance only by the different beam design methods, we here use perfect CSI. In the single stream cases (a), (b), and (c), the proposed algorithm performs as well as the others, except the MAX-SINR algorithm. Also, in these cases the proposed method, redesigning ILS and modified IIA algorithms yield almost the same performance whereas the proposed and redesigning ILS algorithms modified to include signal power perform in-between the MAX-SINR and the other three algorithms. Hence, the modified approach with signal power consideration is a reasonable tradeoff between the sum rate and complexity. In the infeasible case (c), the sum rate saturates after the linear increase w.r.t. SNR due to interference leakage, as expected. In the multistream case (d) with $d=2$, the proposed algorithm performs worse than the IIA method since the MSE of the predictive approach increases with $d$. Next, Fig. 7 shows the average sum rate including channel prediction using the Kalman filter for the case with $M=N=2$, $K=3$, and $d=1$. The rate loss due to imperfect channel information and beam update increases as the mobile speed increases, as expected, and the loss occurs mainly at high SNR. It is seen that the rate loss caused by channel prediction is larger than that by predictive beam design and the redesigning and predictively updating approaches using imperfect CSI show nearly the same performance due to the dominance of channel prediction error. Thus, at high SNR, the predicted channel quality should be also improved to avoid the rate loss in systems operating under interference alignment. As mentioned previously, we used the first-order Gauss-Markov channel model for simple analysis in this paper. By adopting a higher-order channel model and corresponding Kalman filtering, the quality of channel prediction can be improved.

\section{CONCLUSION}

We have proposed a predictive algorithm for the beam design for interference alignment in slowly fading MIMO interference channels based on matrix perturbation theory and newly derived null space update formulas. The proposed algorithm mixes eigen-decomposition and prediction steps properly to minimize complexity while predicting interference-aligning beams effectively in slowly fading channels. We have analyzed the performance of the proposed algorithm to gain insights into the factors affecting its prediction performance and have provided numerical results to validate the proposed algorithm. The algorithm provides an efficient way to track interference-aligning beams with comparable sum rate performance with less complexity than the previous redesigning approaches with timevarying channel information.

\section{APPENDIX}

Proof of Theorem 1 [25]: It is sufficient to show that a full column-rank matrix $\breve{\mathbf{V}}$ (with $d$ columns) and $\left\{\mathbf{A}_{k l}\right\}$ exist such that $\tilde{\mathbf{H}} \breve{\mathbf{V}}=0$. $(\operatorname{rank}(\breve{\mathbf{V}})$ cannot be greater than $d$ by the known 


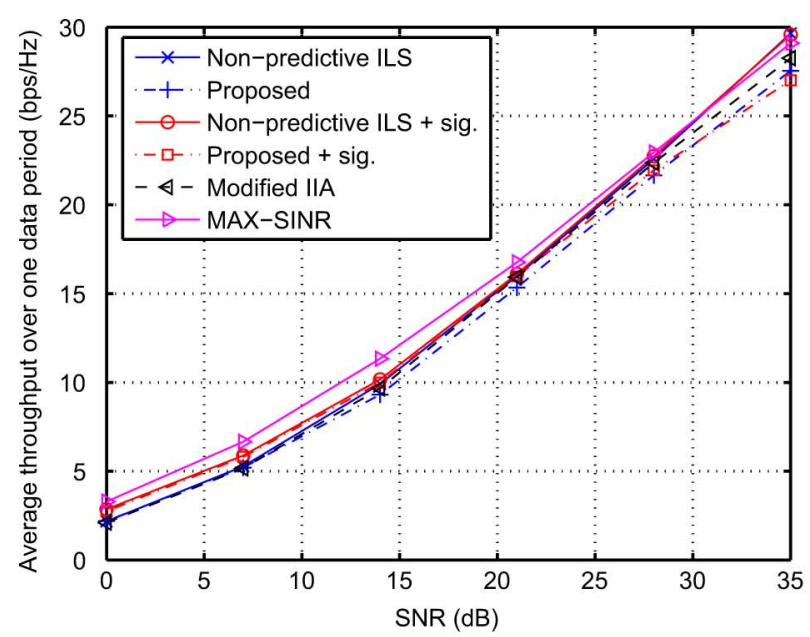

(a)

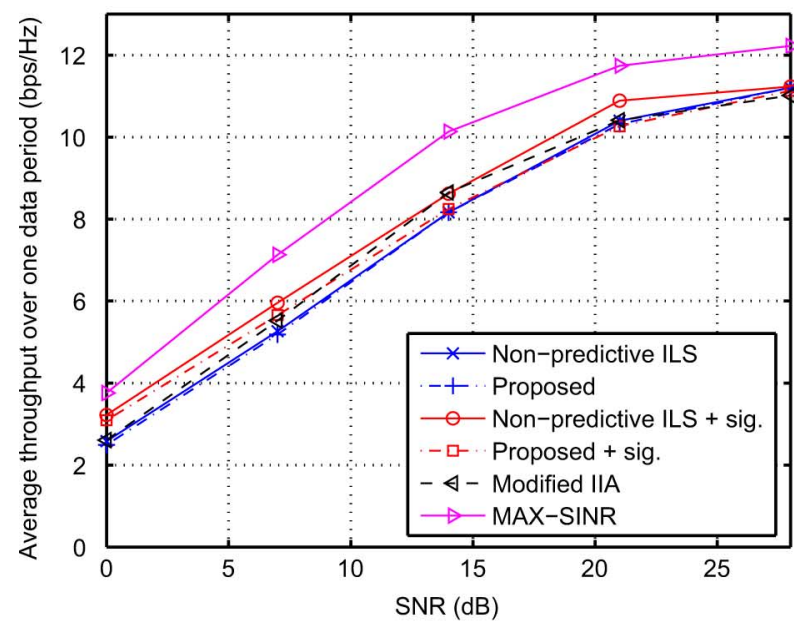

(c)

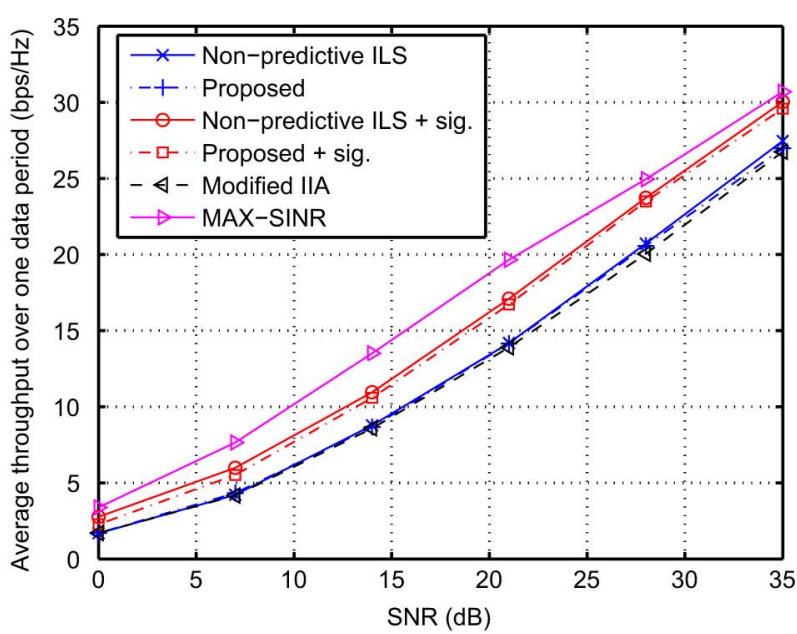

(b)

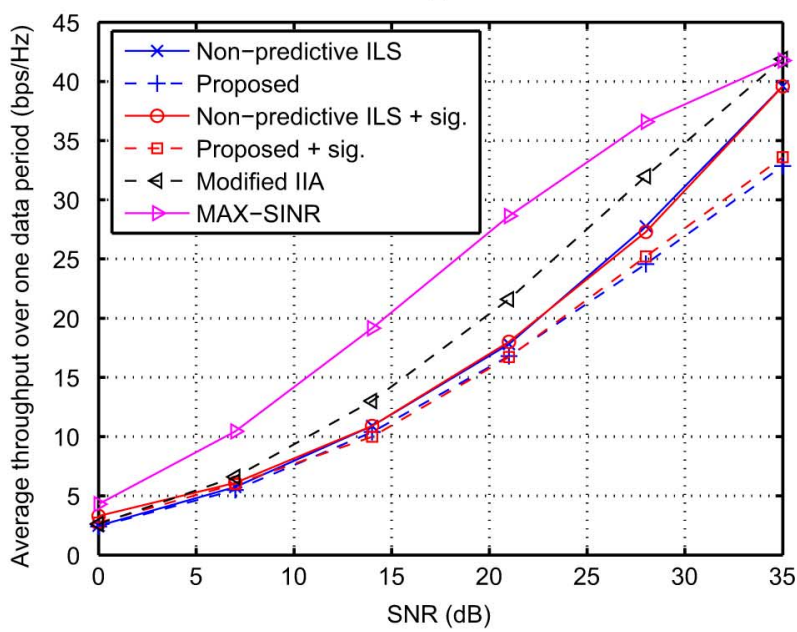

(d)

Fig. 6. Average sum throughput with perfect CSI and $30 \frac{\mathrm{km}}{\mathrm{h}}$ mobile speed (a) $M=N=2, K=3, d=1$. (b) $M=2, N=3, K=3, d=1$. (c) $M=N=2, K=4, d=1$. (d) $M=N=4, K=3, \stackrel{h}{d}=2$.

feasibility result [1].) By partitioning $\breve{\mathbf{V}}$ into $\left[\breve{\mathbf{V}}_{1}^{T}, \breve{\mathbf{V}}_{2}^{T}, \breve{\mathbf{V}}_{3}^{T}\right]^{T}$, $\tilde{\mathbf{H}} \breve{\mathbf{V}}=\mathbf{0}$ can be rewritten as

$$
\begin{aligned}
& \left(\mathbf{I}_{d} \otimes \mathbf{H}_{12}\right) \breve{\mathbf{V}}_{2}-\left(\mathbf{A}_{13} \otimes \mathbf{H}_{13}\right) \breve{\mathbf{V}}_{3}=\mathbf{0} \\
& \left(\mathbf{I}_{d} \otimes \mathbf{H}_{21}\right) \breve{\mathbf{V}}_{1}-\left(\mathbf{A}_{23} \otimes \mathbf{H}_{23}\right) \breve{\mathbf{V}}_{3}=\mathbf{0} \\
& \left(\mathbf{I}_{d} \otimes \mathbf{H}_{31}\right) \breve{\mathbf{V}}_{1}-\left(\mathbf{A}_{32} \otimes \mathbf{H}_{32}\right) \breve{\mathbf{V}}_{2}=\mathbf{0} .
\end{aligned}
$$

For randomly realized channel $\left\{\mathbf{H}_{k l}\right\}$, the matrices are almost surely invertible. From (33) and (34), we express $\breve{\mathbf{V}}_{3}$ and $\breve{\mathbf{V}}_{2}$ in terms of $\breve{\mathbf{V}}_{1}$ and use them in (32). Then, (32) is expressed as $\left(\mathbf{A}_{23} \mathbf{A}_{13}^{-1} \mathbf{A}_{32}^{-1}\right) \otimes\left(\mathbf{H}_{21}^{-1} \mathbf{H}_{23} \mathbf{H}_{13}^{-1} \mathbf{H}_{12} \mathbf{H}_{32}^{-1} \mathbf{H}_{31}\right) \breve{\mathbf{V}}_{1}=\breve{\mathbf{V}}_{1}$ since $(\mathbf{A} \otimes \mathbf{B})^{-1}=\mathbf{A}^{-1} \otimes \mathbf{B}^{-1}$ for invertible matrices $\mathbf{A}$ and $\mathbf{B}$, and $(\mathbf{A} \otimes \mathbf{B})(\mathbf{C} \otimes \mathbf{D})=\mathbf{A C} \otimes \mathbf{B D}$. Defining $\mathbf{A}^{\prime} \triangleq \mathbf{A}_{23} \mathbf{A}_{13}^{-1} \mathbf{A}_{32}^{-1}$ and $\mathbf{F} \triangleq \mathbf{H}_{21}^{-1} \mathbf{H}_{23} \mathbf{H}_{13}^{-1} \mathbf{H}_{12} \mathbf{H}_{32}^{-1} \mathbf{H}_{31}$, we obtain

$$
\left(\mathbf{A}^{\prime} \otimes \mathbf{F}\right) \breve{\mathbf{V}}_{1}=\breve{\mathbf{V}}_{1} \mathbf{I}
$$

Note that (35) is an equation for subspace invariance with $\mathcal{C}\left(\breve{\mathbf{V}}_{1}\right)$ as the invariant subspace of operator $\mathbf{A}^{\prime} \otimes \mathbf{F}$. We denote the sets of eigenvalues and eigenvectors of $\mathbf{A}^{\prime}$ by $\left\{\lambda_{A^{\prime}, 1}, \ldots \lambda_{A^{\prime}, d}\right\}$ and $\left\{\mathbf{a}_{1}, \ldots, \mathbf{a}_{d}\right\}$, respectively, and those of $\mathbf{F}$ by $\left\{\lambda_{F, 1}, \ldots, \lambda_{F, N}\right\}$ and $\left\{\mathbf{f}_{1}, \ldots, \mathbf{f}_{N}\right\}$, respectively. Since the set of complex $N \times N$ matrices that are not diagonalizable has measure zero in the space of $\mathbb{C}^{N \times N}$, the randomly generated matrix $\mathbf{F}$ is almost surely diagonalizable and its $N$ eigenvectors $\left\{\mathbf{f}_{1}, \ldots, \mathbf{f}_{N}\right\}$ are linearly independent. (Also, $\operatorname{Pr}\left\{\lambda_{F, m}=0\right\}=0$.) It is always possible to design $\mathbf{A}^{\prime}$ so that $\mathbf{A}^{\prime}$ has $d$ eigenvalues such that $\lambda_{A^{\prime}, m}=\frac{1}{\lambda_{F, \pi(m)}}$ for some permutation $\pi(\cdot)$. For such an $\mathbf{A}^{\prime}, \mathbf{A}^{\prime} \otimes \mathbf{F}$ has a unit eigenvalue $(\lambda=1)$ with geometric multiplicity $d$, and the corresponding eigenvectors are given by $\left\{\mathbf{a}_{1} \otimes \mathbf{f}_{\pi(1)}, \ldots, \mathbf{a}_{d} \otimes \mathbf{f}_{\pi(d)}\right\}$. These eigenvectors are linearly independent because of the linear independence of $\left\{\mathbf{f}_{\pi(m)}\right\}$. Let $\overrightarrow{\mathbf{V}}_{1}$ be a $d N \times d$ matrix consisting of the eigenvectors $\left\{\mathbf{a}_{m} \otimes \mathbf{f}_{\pi(m)}: m=1, \ldots d\right\}$ as its columns in the form of (13). Then, $\breve{\mathbf{V}}_{1}$ satisfies (35). The expressions for $\breve{\mathbf{V}}_{2}$ and $\breve{\mathbf{V}}_{3}$ can be derived in a similar manner. Finally, the matrix $\breve{\mathbf{V}}$ constructed as such has rank $d$ since the submatrix $\breve{\mathbf{V}}_{1}$ of $\breve{\mathbf{V}}$ has rank $d$.

Proof of Theorem 2: Define $\overline{\mathbf{G}}_{m}[n] \triangleq \frac{1}{\epsilon} \mathbf{G}_{m}[n]$ for $\epsilon \triangleq$ $\left\|\mathbf{G}_{m}[n]\right\|_{2}$. Then, $\mathbf{R}[n]=\mathbf{R}[m]+\epsilon \overline{\mathbf{G}}_{m}[n]$. We denote the 


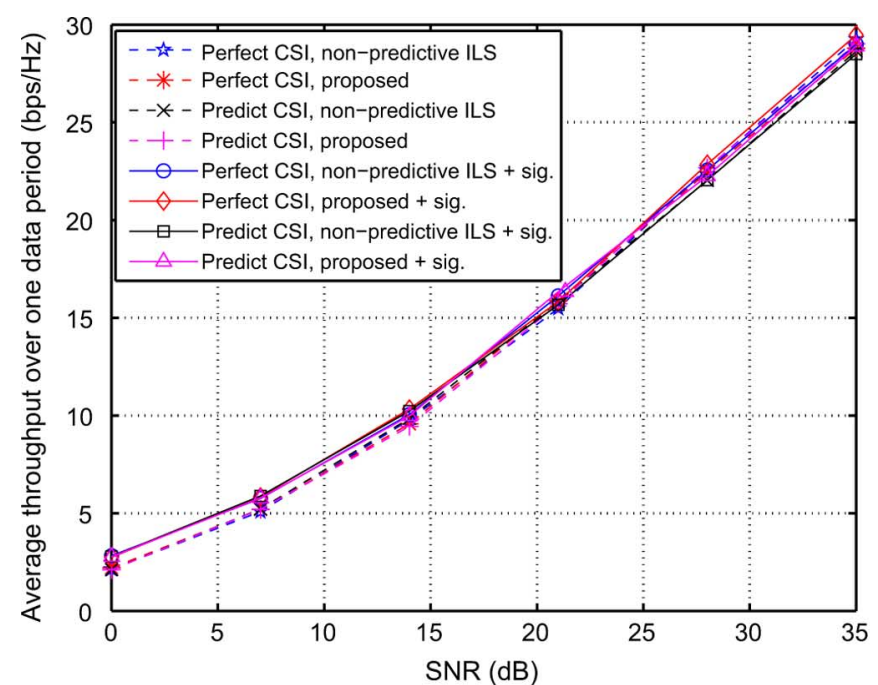

(a)

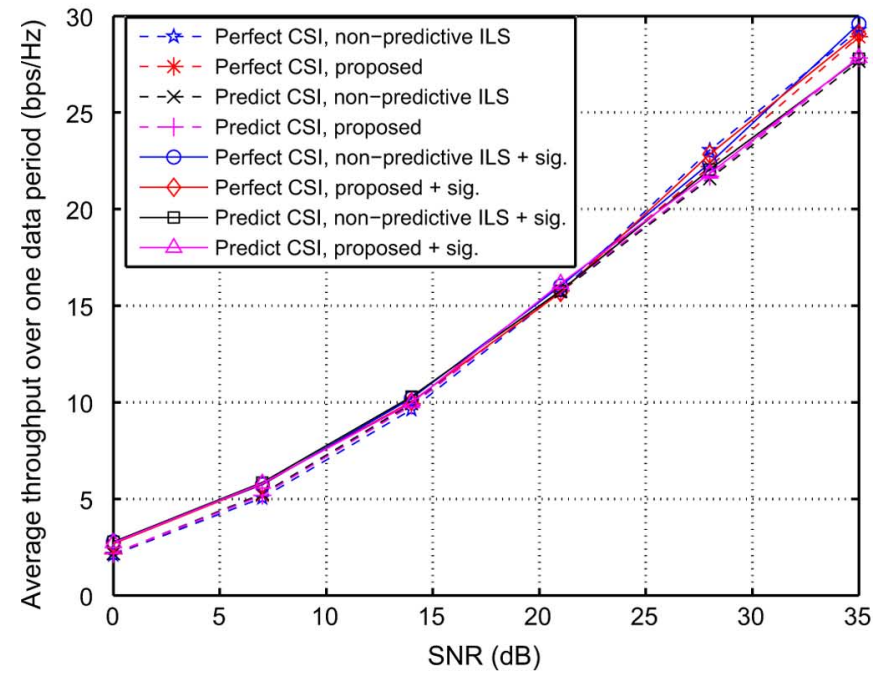

(b)

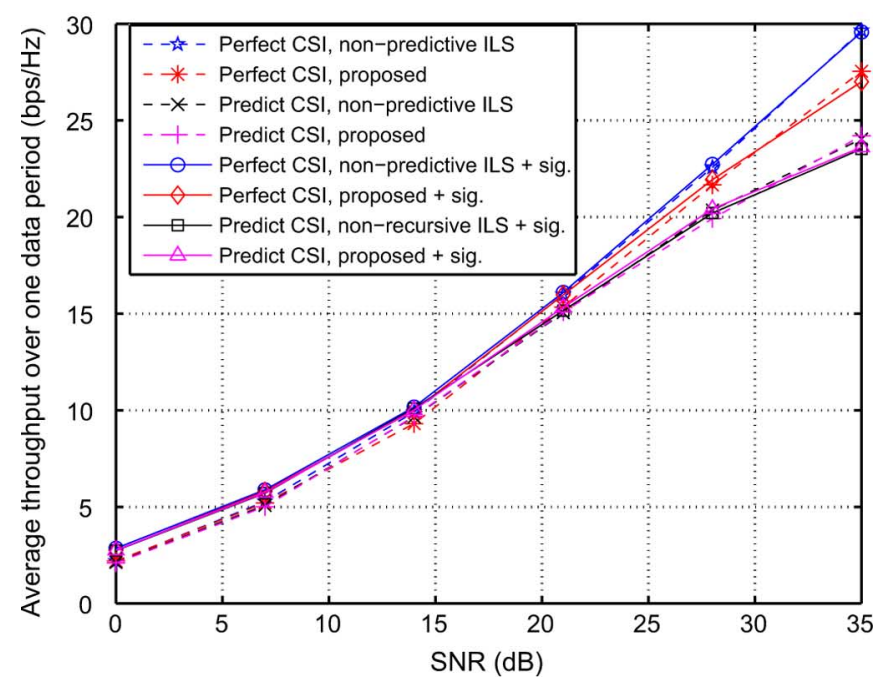

(c)

Fig. 7. Average sum throughput with perfect/predicted CSI and different mobile speeds (a) $M=N=2, K=3, d=1,3 \frac{\mathrm{km}}{\mathrm{h}}$. (b) $M=N=2, K=3$, $d=1,10 \frac{\mathrm{km}}{\mathrm{h}}$. (c) $M=N=2, K=3, d=1,30 \frac{\mathrm{km}}{\mathrm{h}}$. smallest eigenvalue of $\mathbf{R}[n]$ by $\lambda_{1}(\epsilon)$ and the corresponding eigenvector by $\mathbf{v}[n](\epsilon)$. Then

$$
\left(\mathbf{R}[m]+\epsilon \overline{\mathbf{G}}_{m}[n]\right) \mathbf{v}[n](\epsilon)=\lambda_{1}(\epsilon) \mathbf{v}[n](\epsilon) .
$$

Assuming that both $\lambda_{1}(\epsilon)$ and $\mathbf{v}[n](\epsilon)$ are differentiable w.r.t. $\epsilon$, the result in (16) can be obtained by deriving the second-order Taylor expansion of $\mathbf{v}[n](\epsilon)$, which is an extension of the firstorder Taylor expansion in [12, p. 346 ]. By differentiating (36) w.r.t. $\epsilon$, we have

$$
\begin{aligned}
\overline{\mathbf{G}}_{m}[n] \mathbf{v}[n](\epsilon)+\mathbf{R}[m] \dot{\mathbf{v}}[n](\epsilon) \\
+\epsilon \overline{\mathbf{G}}_{m}[n] \dot{\mathbf{v}}[n](\epsilon)=\dot{\lambda}_{1}(\epsilon) \mathbf{v}[n](\epsilon)+\lambda_{1}(\epsilon) \dot{\mathbf{v}}[n](\epsilon)
\end{aligned}
$$

Differentiating the above equation w.r.t. $\epsilon$ again and setting $\epsilon=$ 0 yields

$$
\begin{aligned}
2 \overline{\mathbf{G}}_{m}[n] \dot{\mathbf{v}}[n](0)+\mathbf{R}[m] \ddot{\mathbf{v}}[n](0) \\
=\ddot{\lambda}_{1}(0) \mathbf{v}[n](0)+2 \dot{\lambda}_{1}(0) \dot{\mathbf{v}}[n](0)+\lambda_{1} \ddot{\mathbf{v}}[n](0)
\end{aligned}
$$

Since the eigenvectors $\mathbf{q}_{1}, \mathbf{q}_{2}, \ldots, \mathbf{q}_{r}$ of $\mathbf{R}[m]$ form a basis in $\mathbb{C}^{r}$ in the full rank case, $\dot{\mathbf{v}}[n](0)$ and $\ddot{\mathbf{v}}[n](0)$ are represented as

$$
\dot{\mathbf{v}}[n](0)=\sum_{i=1}^{r_{F}} a_{i} \mathbf{q}_{i}
$$

and

$$
\ddot{\mathbf{v}}[n](0)=\sum_{i=1}^{r_{F}} b_{i} \mathbf{q}_{i}
$$

respectively. From $[12, \quad$ p. 346], $\dot{\mathbf{v}}[n](0)=$ $\sum_{i=2}^{r_{F}} \frac{\mathbf{q}_{i}^{H} \overline{\mathbf{G}}_{m}[n] \mathbf{q}_{1}}{\left(\lambda_{1}-\lambda_{i}\right)} \mathbf{q}_{i}$. Hence

$$
a_{i}= \begin{cases}0, & \text { for } i=1, \\ \frac{\mathbf{q}_{i}^{H} \overline{\mathbf{G}}_{m}[n] \mathbf{v}[m]}{\lambda_{1}-\lambda_{i}}, & \text { for } i=2, \ldots, r_{F}, .\end{cases}
$$

where $\mathbf{v}[m]=\mathbf{q}_{1}$. Thus, (38) is rewritten as

$$
\begin{aligned}
2 \overline{\mathbf{G}}_{m}[n] & \sum_{i=2}^{r_{F}} a_{i} \mathbf{q}_{i}+\mathbf{R}[m] \sum_{i=1}^{r_{F}} b_{i} \mathbf{q}_{i} \\
& =\ddot{\lambda}_{1}(0) \mathbf{q}_{1}+2 \dot{\lambda}_{1}(0) \sum_{i=2}^{r_{F}} a_{i} \mathbf{q}_{i}+\lambda_{1}(0) \sum_{i=1}^{r_{F}} b_{i} \mathbf{q}_{i} .
\end{aligned}
$$

Since $\mathbf{R}[m] \mathbf{q}_{i}=\lambda_{i}(0) \mathbf{q}_{i}$ and $\mathbf{v}[n](0)=\mathbf{q}_{1}$, (42) becomes

$$
\begin{gathered}
\ddot{\lambda}_{1}(0) \mathbf{q}_{1}=2 \overline{\mathbf{G}}_{m}[n] \sum_{i=2}^{r_{F}} a_{i} \mathbf{q}_{i}-2 \dot{\lambda}_{1}(0) \sum_{i=2}^{r_{F}} a_{i} \mathbf{q}_{i} \\
+\sum_{i=2}^{r_{F}} b_{i}\left(\lambda_{i}(0)-\lambda_{1}(0)\right) \mathbf{q}_{i} .
\end{gathered}
$$

Multiplying $\mathbf{q}_{j}^{H}$ to (43) from the left yields

$$
b_{j}=\frac{2}{\left(\lambda_{1}-\lambda_{j}\right)}\left(\sum_{i=2}^{r_{F}} a_{i} \mathbf{q}_{j}^{H} \overline{\mathbf{G}}_{m}[n] \mathbf{q}_{i}-\dot{\lambda}_{1}(0) a_{j}\right)
$$


where $\lambda_{i}=\lambda_{i}(0)$ for $i=1, \ldots, r_{F}$. To evaluate $\dot{\lambda}_{1}(0)$, we set $\epsilon=0$ in (37) and multiply the equation by $\mathbf{v}[n]^{H}(0)$. Then

$$
\begin{aligned}
\mathbf{q}_{1}^{H} \overline{\mathbf{G}}_{m}[n] \mathbf{q}_{1}+\mathbf{q}_{1}^{H} \mathbf{R}[m] & \dot{\mathbf{v}}[n](0) \\
& =\dot{\lambda}_{1}(0) \mathbf{q}_{1}^{H} \mathbf{q}_{1}+\mathbf{q}_{1}^{H} \lambda_{1}(0) \dot{\mathbf{v}}[n](0)
\end{aligned}
$$

where $\mathbf{q}_{1}=\mathbf{v}[n](0)$. Since $\mathbf{R}[m]$ is a symmetric matrix, $\mathbf{q}_{1}^{H} \mathbf{R}[m]=\lambda_{1}(0) \mathbf{q}_{1}^{H}$, and (45) reduces to

$$
\dot{\lambda}_{1}(0)=\mathbf{q}_{1}^{H} \overline{\mathbf{G}}_{m}[n] \mathbf{q}_{1} \text {. }
$$

From (39), (40), and (44), the Taylor expansion for $\mathbf{v}[n](\epsilon)$ is given by

$$
\begin{aligned}
\mathbf{v}[n](\epsilon)= & \mathbf{v}[n](0)+\epsilon \dot{\mathbf{v}}[n](0)+\frac{\epsilon^{2}}{2} \ddot{\mathbf{v}}[n](0)+\mathcal{O}\left(\epsilon^{3}\right) \\
= & \mathbf{v}[m]-\epsilon \sum_{i=2}^{r_{F}} \frac{\mathbf{q}_{i}^{H} \overline{\mathbf{G}}_{m}[n] \mathbf{v}[m]}{\lambda_{i}-\lambda_{1}} \mathbf{q}_{i} \\
& +\frac{\epsilon^{2}}{2} \sum_{j=2}^{r_{F}} \frac{2}{\left(\lambda_{1}-\lambda_{j}\right)} \\
& \times\left(\sum_{i=2}^{r_{F}} a_{i} \mathbf{q}_{j}^{H} \overline{\mathbf{G}}_{m}[n] \mathbf{q}_{i}-\dot{\lambda}_{1}(0) a_{j}\right) \mathbf{q}_{j} \\
& +O\left(\epsilon^{3}\right)
\end{aligned}
$$

The desired result in (16) is obtained from (46) because $O\left(\epsilon^{3}\right)$ implies $o\left(\epsilon^{2}\right)$.

Theorem A.1 (Avrachenkov and Haviv, 2003): Suppose that $\mathbf{R}(\epsilon)$ is a rank-preserving perturbation of $\mathbf{R}_{0}$; i.e., $\mathbf{R}(\epsilon)$ and $\mathbf{R}_{0}$ have the same rank and $\mathbf{R}(\epsilon)=\mathbf{R}_{0}+\epsilon \mathbf{R}_{1}+\epsilon^{2} \mathbf{R}_{2}+\cdots$. Then, there exists a holomorphic family of eigenvectors, $\boldsymbol{\Theta}(\epsilon) \triangleq \boldsymbol{\Theta}_{0}+\epsilon \boldsymbol{\Theta}_{1}+\epsilon^{2} \boldsymbol{\Theta}_{2}+\cdots$, corresponding to the zero eigenvalue and satisfying the normalization condition $\boldsymbol{\Theta}_{0}^{H} \boldsymbol{\Theta}(\epsilon)=\mathbf{I}$, where $\boldsymbol{\Theta}_{0}=\tilde{\boldsymbol{\Theta}}$ such that $\mathbf{R}_{0} \tilde{\boldsymbol{\Theta}}=\mathbf{0}$ and $\tilde{\boldsymbol{\Theta}}^{H} \tilde{\boldsymbol{\Theta}}=\mathbf{I}$. The coefficients of the power series for $\boldsymbol{\Theta}(\epsilon)$ can be calculated recursively by the formula

$$
\boldsymbol{\Theta}_{k}=-\mathbf{R}_{0}^{\dagger} \sum_{j=1}^{k} \mathbf{R}_{j} \boldsymbol{\Theta}_{k-j}, k=1,2, \ldots
$$

Proof of Theorem 3: To prove Theorem 3, we need the above result regarding the perturbation of a null space in [32].

Theorem 3 can be proved by considering the first-order approximation of $\mathbf{R}(\epsilon)$ and the second-order approximation of $\boldsymbol{\Theta}(\epsilon)$ in Theorem A.1. Specifically, we write $\mathbf{R}[n]=\mathbf{R}[m]+$ $\epsilon \overline{\mathbf{G}}_{m}[n]$ where $\overline{\mathbf{G}}_{m}[n]=\frac{\mathbf{G}_{m}[n]}{\epsilon}$ and $\epsilon=\left\|\mathbf{G}_{m}[n]\right\|_{2}$, and consider the eigen-decompositions of $\mathbf{R}[m]$ and $\mathbf{R}[n]$

$$
\begin{aligned}
\mathbf{R}[m]= & {\left[\begin{array}{ll}
\mathbf{Q}_{r} & \tilde{\mathbf{Q}}_{r}
\end{array}\right] \operatorname{diag}\left(\lambda_{1}, \ldots, \lambda_{r}, 0, \ldots, 0\right) } \\
& \times\left[\begin{array}{ll}
\mathbf{Q}_{r} & \tilde{\mathbf{Q}}_{r}
\end{array}\right]^{H}
\end{aligned}
$$

and

$$
\begin{aligned}
\mathbf{R}[n]= & {\left[\begin{array}{ll}
\mathbf{Q}_{r}(\epsilon) & \tilde{\mathbf{Q}}_{r}(\epsilon)
\end{array}\right] } \\
& \times \operatorname{diag}\left(\lambda_{1}(\epsilon), \ldots, \lambda_{r}(\epsilon), 0, \ldots, 0\right) \\
& \times\left[\begin{array}{ll}
\mathbf{Q}_{r}(\epsilon) & \tilde{\mathbf{Q}}_{r}(\epsilon)
\end{array}\right]^{H}
\end{aligned}
$$

where $\mathbf{Q}_{r}=\left[\begin{array}{lll}\mathbf{q}_{1} & \cdots & \mathbf{q}_{r}\end{array}\right] ; \tilde{\mathbf{Q}}_{r}$ is a submatrix of the eigenvector matrix of $\mathbf{R}[m]$ corresponding to the zero eigenvalues; $\mathbf{Q}_{r}(\epsilon)$ and $\tilde{\mathbf{Q}}_{r}(\epsilon)$ are submatrices of the eigenvector matrix of $\mathbf{R}[n]$, which are perturbed versions of $\mathbf{Q}_{r}$ and $\tilde{\mathbf{Q}}_{r}$, respectively.

$\mathbf{R}[n]$ is a rank-preserving perturbation of $\mathbf{R}[m]$ because the rank of $\tilde{\mathbf{H}}[n]$ is almost surely identical to that of $\tilde{\mathbf{H}}[\mathrm{m}]$. Therefore, $\tilde{\mathbf{Q}}_{r}(\epsilon)$ can be written as

$$
\tilde{\mathbf{Q}}_{r}(\epsilon)=\tilde{\mathbf{Q}}_{r}+\epsilon \boldsymbol{\Theta}_{1}+\epsilon^{2} \boldsymbol{\Theta}_{2}+O\left(\epsilon^{3}\right)
$$

where $\boldsymbol{\Theta}_{1}$ and $\boldsymbol{\Theta}_{2}$ are obtained from (47)

$$
\boldsymbol{\Theta}_{1}=-\mathbf{R}[m]^{\dagger} \overline{\mathbf{G}}_{m}[n] \tilde{\mathbf{Q}}_{r}, \boldsymbol{\Theta}_{2}=-\mathbf{R}[m]^{\dagger} \overline{\mathbf{G}}_{m}[n] \boldsymbol{\Theta}_{1} .
$$

[The following relations are used in (47): $\mathbf{R}_{0}=\mathbf{R}[m]$, $\mathbf{R}_{1}=\overline{\mathbf{G}}_{m}[n]$ and $\mathbf{R}_{j}=0$ for $j=2, \ldots, k$.] Since $\mathbf{R}[m]^{\dagger}=\sum_{i=1}^{r} \frac{1}{\lambda_{i}} \mathbf{q}_{i} \mathbf{q}_{i}^{H}, \boldsymbol{\Theta}_{1}$ and $\boldsymbol{\Theta}_{2}$ are rewritten as

$$
\begin{aligned}
& \boldsymbol{\Theta}_{1}=-\sum_{i=1}^{r} \frac{1}{\lambda_{i}} \mathbf{q}_{i} \mathbf{q}_{i}^{H} \overline{\mathbf{G}}_{m}[n] \tilde{\mathbf{Q}}_{r} \\
& \boldsymbol{\Theta}_{2}=\sum_{i=1}^{r} \frac{1}{\lambda_{i}} \mathbf{q}_{i} \mathbf{q}_{i}^{H} \overline{\mathbf{G}}_{m}[n]\left(\sum_{i=1}^{r} \frac{1}{\lambda_{i}} \mathbf{q}_{i} \mathbf{q}_{i}^{H} \overline{\mathbf{G}}_{m}[n] \tilde{\mathbf{Q}}_{r}\right) .
\end{aligned}
$$

From (48), we have

$$
\begin{aligned}
\tilde{\mathbf{Q}}_{r}(\epsilon)= & \tilde{\mathbf{Q}}_{r}-\epsilon \sum_{i=1}^{r} \frac{1}{\lambda_{i}} \mathbf{q}_{i} \mathbf{q}_{i}^{H} \overline{\mathbf{G}}_{m}[n] \tilde{\mathbf{Q}}_{r} \\
& +\epsilon^{2} \sum_{i=1}^{r} \frac{1}{\lambda_{i}} \mathbf{q}_{i} \mathbf{q}_{i}^{H} \overline{\mathbf{G}}_{m}[n] \\
& \times\left(\sum_{i=1}^{r} \frac{1}{\lambda_{i}} \mathbf{q}_{i} \mathbf{q}_{i}^{H} \overline{\mathbf{G}}_{m}[n] \tilde{\mathbf{Q}}_{r}\right)+O\left(\epsilon^{3}\right) .
\end{aligned}
$$

The interference aligning beam vectors $\mathbf{v}[m]$ and $\mathbf{v}[n]$ are given by linear combinations of the columns of $\tilde{\mathbf{Q}}_{r}$ and $\tilde{\mathbf{Q}}_{r}(\epsilon)$, respectively; i.e., $\mathbf{v}[m]=\tilde{\mathbf{Q}}_{r} \boldsymbol{\mu}$ and $\mathbf{v}[n]=\tilde{\mathbf{Q}}_{r}(\epsilon) \boldsymbol{\mu}$ for some constant vector $\boldsymbol{\mu}$. Multiplying $\boldsymbol{\mu}$ to (49) from the right, we have

$$
\begin{aligned}
\mathbf{v}[n]= & \mathbf{v}[m]-\epsilon \sum_{i=1}^{r} \frac{\mathbf{q}_{i} \mathbf{q}_{i}^{H} \overline{\mathbf{G}}_{m}[n] \mathbf{v}[m]}{\lambda_{i}} \\
& +\epsilon^{2} \sum_{i=1}^{r} \frac{1}{\lambda_{i}} \mathbf{q}_{i} \mathbf{q}_{i}^{H} \overline{\mathbf{G}}_{m}[n] \\
& \times\left(\sum_{i=1}^{r} \frac{1}{\lambda_{i}} \mathbf{q}_{i} \mathbf{q}_{i}^{H} \overline{\mathbf{G}}_{m}[n] \mathbf{v}[m]\right)+O\left(\epsilon^{3}\right) \\
= & \mathbf{v}[m]-\epsilon \sum_{i=1}^{r} \frac{\mathbf{q}_{i}^{H} \overline{\mathbf{G}}_{m}[n] \mathbf{v}[m]}{\lambda_{i}} \mathbf{q}_{i} \\
& +\epsilon^{2} \sum_{i=1}^{r} \frac{1}{\lambda_{i}} \mathbf{q}_{i} \mathbf{q}_{i}^{H} \overline{\mathbf{G}}_{m}[n] \\
& \times\left(\sum_{i=1}^{r} \frac{1}{\lambda_{i}} \mathbf{q}_{i}^{H} \overline{\mathbf{G}}_{m}[n] \mathbf{v}[m] \mathbf{q}\right)+O\left(\epsilon^{3}\right) \\
= & \mathbf{v}[m]-\epsilon \sum_{i=1}^{r} \frac{\mathbf{q}_{i}^{H} \overline{\mathbf{G}}_{m}[n] \mathbf{v}[m]}{\lambda_{i}} \mathbf{q}_{i} \\
& +\epsilon^{2} \sum_{j=1}^{r} \frac{1}{\lambda_{j}}\left(\sum_{i=1}^{r} a_{i} \mathbf{q}_{j}^{H} \overline{\mathbf{G}}_{m}[n] \mathbf{q}_{i}\right) \mathbf{q}_{j}+O\left(\epsilon^{3}\right)
\end{aligned}
$$

where the second equality holds because $\mathbf{q}_{i}^{H} \mathbf{G}_{m}[n] \mathbf{v}[m]$ is a scalar and $a_{i} \triangleq \frac{\mathbf{q}_{i}^{H} \mathbf{G}_{m}[n] \mathbf{v}[m]}{\lambda_{i}}$. 
Proof of Theorem 4: Using the definition of $\hat{\mathbf{G}}_{m}[m+p]$ and $\hat{\mathbf{H}}_{k l}[m+p]=\beta_{k l}^{p} \hat{\mathbf{H}}_{k l}[m]$, we have

$$
\begin{aligned}
\| \hat{\mathbf{G}}_{m} & {[m+p] \|_{2} } \\
= & \underset{\hat{\mathbf{H}}}{\tilde{y}}[m+p]^{H} \tilde{\hat{\mathbf{H}}}[m+p]-\tilde{\hat{\mathbf{H}}}[m]^{H} \tilde{\hat{\mathbf{H}}}[m] \\
= & \|(\tilde{\mathbf{B}}[p] \circ \tilde{\hat{\mathbf{H}}}[m])^{H}(\tilde{\mathbf{B}}[p] \circ \tilde{\hat{\mathbf{H}}}[m]) \\
& -\tilde{\hat{\mathbf{H}}}[m]^{H} \tilde{\hat{\mathbf{H}}}[m] \|_{2} \\
\leq & \operatorname{tr}\left\{\tilde{\hat{\mathbf{H}}}[m]^{H} \tilde{\hat{\mathbf{H}}}[m]\right. \\
& \left.-(\tilde{\mathbf{B}}[p] \circ \tilde{\hat{\mathbf{H}}}[m])^{H}(\tilde{\mathbf{B}}[p] \circ \tilde{\hat{\mathbf{H}}}[m])\right\} \\
= & \sum_{i, j}\left(1-[\tilde{\mathbf{B}}[p]]_{i j}^{2}\right)\left|[\tilde{\hat{\mathbf{H}}}[m]]_{i j}\right|^{2},
\end{aligned}
$$

where $\tilde{\mathbf{B}}[p]$ is defined below. In the second equality, we use the following relations in (52), shown at the bottom of the page. The inequality holds since $\|\mathbf{M}\|_{2}=\lambda_{\max }(\mathbf{M}) \leq \sum_{i} \lambda_{i}(\mathbf{M})=$ $\operatorname{tr}(\mathbf{M})$. Under the assumption of $\beta_{k l}=\beta$ for all $k$ and $l$, we have

$$
\left\|\hat{\mathbf{G}}_{m}[m+p]\right\|_{2} \leq\left(1-\beta^{2 p}\right) \sum_{i, j}\left|[\tilde{\hat{\mathbf{H}}}[m]]_{i j}\right|^{2} .
$$

By taking expectation w.r.t. channel distribution, we have

$$
\begin{aligned}
& \mathbb{E}\left\|\hat{\mathbf{G}}_{m}[m+p]\right\|_{2} \\
& \quad \leq\left(1-\beta^{2 p}\right)\left(1+\sqrt{6 \sigma_{a}^{4}} d\right) \Pi_{0}^{2} K(K-2) M N d .
\end{aligned}
$$

In $\tilde{\hat{\mathbf{H}}}[m]$, two different kinds of nonzero submatrices exist. Some submatrices are Kronecker products of a channel matrix and an identity matrix, and the others are those of a channel matrix and a linear combining matrix $\mathbf{A}_{k l}$. The numbers of elements corresponding to the former and later cases are

$$
\begin{aligned}
& \tilde{\hat{\mathbf{H}}}[m+p]=\left[\begin{array}{cccccc}
\mathbf{0} & \mathbf{I}_{d} \otimes \hat{\mathbf{H}}_{12}[m+p] & -\mathbf{A}_{13} \otimes \hat{\mathbf{H}}_{13}[m+p] & \mathbf{0} & \cdots & \mathbf{0} \\
\mathbf{0} & \mathbf{I}_{d} \otimes \hat{\mathbf{H}}_{12}[m+p] & \mathbf{0} & -\mathbf{A}_{14} \otimes \hat{\mathbf{H}}_{14}[m+p] & \cdots & \mathbf{0} \\
\vdots & \vdots & \vdots & \vdots & \vdots & \vdots \\
\mathbf{I}_{d} \otimes \hat{\mathbf{H}}_{K 1}[m+p] & \mathbf{0} & \cdots & \mathbf{0} & \cdots & \mathbf{0}
\end{array}\right] \\
& =\left[\begin{array}{cccccc}
\mathbf{0} & \mathbf{I}_{d} \otimes \beta_{12}^{p} \hat{\mathbf{H}}_{12}[m] & -\mathbf{A}_{13} \otimes \beta_{13}^{p} \hat{\mathbf{H}}_{13}[m] & \mathbf{0} & \cdots & \mathbf{0} \\
\mathbf{0} & \mathbf{I}_{d} \otimes \beta_{12}^{p} \hat{\mathbf{H}}_{12}[m] & \mathbf{0} & -\mathbf{A}_{14} \otimes \beta_{14}^{p} \hat{\mathbf{H}}_{14}[m] & \cdots & \mathbf{0} \\
\vdots & \vdots & \vdots & \vdots & \vdots & \vdots \\
\mathbf{I}_{d} \otimes \beta_{K 1}^{p} \hat{\mathbf{H}}_{K 1}[m] & \mathbf{0} & \cdots & \mathbf{0} & \cdots & \mathbf{0}
\end{array}\right] \\
& =\underbrace{\left[\begin{array}{cccccc}
\mathbf{0} & \mathbf{I}_{d} \otimes \beta_{12}^{p} \mathbf{1 1}^{T} & \mathbf{I} \otimes \beta_{13}^{p} \mathbf{1 1}^{T} & \mathbf{0} & \cdots & \mathbf{0} \\
\mathbf{0} & \mathbf{I}_{d} \otimes \beta_{12}^{p} \mathbf{1 1}^{T} & \mathbf{0} & \mathbf{I} \otimes \beta_{14}^{p} \mathbf{1 1}^{T} & \cdots & \mathbf{0} \\
\vdots & \vdots & \vdots & \vdots & \vdots & \vdots \\
\mathbf{I}_{d} \otimes \beta_{K 1}^{p} \mathbf{1 1}^{T} & \mathbf{0} & \cdots & \mathbf{0} & \cdots & \mathbf{0}
\end{array}\right]}_{=: \tilde{\mathbf{B}}[p]} \tilde{\hat{\mathbf{H}}}[m] .
\end{aligned}
$$

$$
\begin{aligned}
\Gamma & \triangleq \mathbb{E} \sum_{k=1}^{K} \log \left(1+\frac{\left|\mathbf{u}_{k}^{H} \mathbf{H}_{k k} \mathbf{v}\right|^{2}}{\sigma^{2}}\right)-\mathbb{E} \sum_{k=1}^{K} \log \left(1+\frac{\mid \hat{\mathbf{u}}_{k}^{H} \mathbf{H}}{\left.\mathbf{H}_{k k} \overline{\mathbf{v}}\right|^{2}}\right) \\
& =\mathbb{E} \sum_{k=1}^{K} \log \left(1+\frac{\left|\mathbf{u}_{k}^{H} \mathbf{H}_{k k} \mathbf{v}\right|^{2}}{\sigma^{2}}\right)-\mathbb{E} \sum_{k=1}^{K} \log \left(1+\frac{\left|\hat{\mathbf{u}}_{k}^{H} \mathbf{H}_{k k} \overline{\mathbf{v}}\right|^{2}+I_{h, k}+I_{v, k}}{\sigma^{2}}\right)+\mathbb{E} \sum_{k=1}^{K} \log \left(1+\frac{I_{h, k}+I_{v, k}}{\sigma^{2}}\right) \\
& \stackrel{(a)}{\leq} \mathbb{E} \sum_{k=1}^{K} \log \left(1+\frac{\left|\mathbf{u}_{k}^{H} \mathbf{H}_{k k} \mathbf{v}\right|^{2}}{\sigma^{2}}\right)-\mathbb{E} \sum_{k=1}^{K} \log \left(1+\frac{\left|\hat{\mathbf{u}}_{k}^{H} \mathbf{H}_{k k} \overline{\mathbf{v}}\right|^{2}}{\sigma^{2}}\right)+\mathbb{E} \sum_{k=1}^{K} \log \left(1+\frac{I_{h, k}+I_{v, k}}{\sigma^{2}}\right) \\
& \stackrel{(b)}{=} \mathbb{E} \sum_{k=1}^{K} \log \left(1+\frac{I_{h, k}+I_{v, k}}{\sigma^{2}}\right) \\
& \stackrel{(c)}{\leq} \sum_{k=1}^{K} \log \left(1+\frac{\mathbb{E}\left\{I_{h, k}\right\}+\mathbb{E}\left\{I_{v, k}\right\}}{\sigma^{2}}\right)
\end{aligned}
$$


$K(K-2) M N d$ and $K(K-2) M N d^{2}$, respectively. Additionally, we use

$$
\begin{aligned}
\mathbb{E}\left\{\left[\mathbf{A}_{k l}\right]_{i^{\prime} j^{\prime}}^{2}\left[\hat{\mathbf{H}}_{k l}\right]_{i j}^{2}\right\} & \leq \sqrt{\mathbb{E}\left\{\left[\mathbf{A}_{k l}\right]_{i^{\prime} j^{\prime}}^{4}\right\} \mathbb{E}\left\{\left[\hat{\mathbf{H}}_{k l}\right]_{i j}^{4}\right\}} \\
& \leq \sqrt{\sigma_{a}^{4} 6 \Pi_{0}^{4}}
\end{aligned}
$$

where $\sigma_{a}^{4}$ is the maximum of the fourth moment of $\left[\mathbf{A}_{k l}\right]_{i^{\prime} j^{\prime}}$. The first inequality is the Cauchy-Schwartz inequality, and the second inequality holds because $\hat{H}_{k l}^{i j} \sim \mathcal{C N}\left(0, \hat{\Pi}_{0}\right)$ and $\hat{\Pi}_{0} \leq$ $\Pi_{0}$,

Proof of Theorem 5: The sum rate loss is given by (55) at the bottom of the previous page, where $I_{h, k}=$ $\sum_{l=1, l \neq k}^{K}\left|\hat{\mathbf{u}}_{k}^{H} \check{\mathbf{H}}_{k l} \overline{\mathbf{v}}_{l}\right|^{2}$ and $I_{v, k}=\sum_{l=1}^{K}\left|\hat{\mathbf{u}}_{k}^{H} \mathbf{H}_{k l} \check{\mathbf{v}}_{l}\right|^{2}[38]$. (a) is by $I_{h, k} \geq 0$ and $I_{v, k} \geq 0$. (b) holds because $\mathbf{v}_{k}, \overline{\mathbf{v}}_{k}$, $\mathbf{u}_{k}$, and $\hat{\mathbf{u}}_{k}$ are isotropically distributed unit vectors and are independent of $\mathbf{H}_{k k}$ [38]. (c) is by Jensen's inequality and the concavity of logarithm. Applying Jensen's inequality again to the last equation, we have

$$
\begin{aligned}
\mathbb{E}\left\{I_{h, k}\right\} & =\mathbb{E} \sum_{l=1, l \neq k}^{K}\left|\hat{\mathbf{u}}_{k}^{H} \check{\mathbf{H}}_{k l} \overline{\mathbf{v}}_{l}\right|^{2} \\
& \leq \sum_{l=1, l \neq k}^{K} \mathbb{E}\left\|\check{\mathbf{H}}_{k l}\right\|_{F}^{2}=(K-1) M N \sigma_{h}^{2}
\end{aligned}
$$

and

$$
\begin{aligned}
\mathbb{E}\left\{I_{v, k}\right\} & =\mathbb{E} \sum_{l=1}^{K}\left|\hat{\mathbf{u}}_{k}^{H} \mathbf{H}_{k l} \check{\mathbf{v}}_{l}\right|^{2} \\
& \leq \sum_{l=1}^{K} \mathbb{E}\left\|\mathbf{H}_{k l}\right\|_{F}^{2}\left|\check{\mathbf{v}}_{l}\right|^{2}=K M N \Pi_{0}^{2} \sigma_{v}^{2}
\end{aligned}
$$

since $\hat{\mathbf{u}}_{k}$ and $\overline{\mathbf{v}}_{l}$ are unit-norm vectors and $\|\mathbf{M}\|_{2} \leq\|\mathbf{M}\|_{F}$ for any matrix $\mathbf{M}$.

\section{REFERENCES}

[1] V. R. Cadambe and S. A. Jafar, "Interference alignment and degrees of freedom of the $K$-user interference channel," IEEE Trans. Inf. Theory, vol. 54, pp. 3425-3441, Aug. 2008.

[2] T. Gou and S. A. Jafar, "Degrees of freedom of the $K$ user $M \times N$ MIMO interference channel," IEEE Trans. Inf. Theory, vol. 56, pp. 6040-6057, Dec. 2010.

[3] R. Erkin and E. Ordentlich, "On the degrees-of-freedom for the $K$-user Gaussian interference channel is discontinuous at rational channel coefficients," IEEE Trans. Inf. Theory, vol. 55, pp. 4932-4946, Nov. 2009.

[4] A. S. Motahari, S. Oveis-Gharan, M. A. Maddah-Ali, and A. K. Khandani, "real Interference Alignment: Exploiting the Potential of Single Antenna Systems," 2009 [Online]. Available: ArXiv pre-print cs.IT/ 0908.2282

[5] C. M. Yetis, T. Gou, S. A. Jafar, and A. H. Kayran, "On feasibility of interference alignment in MIMO interference networks," IEEE Trans. Signal Process., vol. 58, pp. 4771-4782, Sep. 2010.

[6] A. Ghasemi, A. S. Motahari, and A. K. Khandani, "Interference alignment for the $K$ user MIMO interference channel," in Proc. ISIT, Austin, TX, Jun. 2010.

[7] B. Nourani, S. A. Motahari, and A. K. Khandani, "Relay-aided interference alignment for the quasi-static interference channel," in Proc. ISIT, Austin, TX, Jun. 2010.
[8] K. Gomadam, V. R. Cadambe, and S. A. Jafar, “A distributed numerical approach to interference alignment and applications to wireless interference networks," IEEE Trans. Inf. Theory, vol. 57, pp. 3309-3322, Jun. 2011.

[9] H. Yu and Y. Sung, "Least squares approach to joint beam design for interference alignment in multiuser multiinput multioutput interference channels," IEEE Trans. Signal Process., vol. 58, pp. 4960-4966, Sep. 2010.

[10] S. W. Peters and R. W. Heath, "Interference alignment via alternating minimization," in Proc. ICASSP, Taipei, Taiwan, Apr. 2009.

[11] H. Yu, Y. Sung, H. Kim, and Y. H. Lee, "Adaptive beam tracking for interference alignment for multiuser time-varying MIMO interference channels," in Proc. ICASSP, Dallas, TX, Mar. 2010.

[12] G. H. Golub and C. F. V. Loan, Matrix Computations, 2nd ed. Baltimore, MD: Johns Hopkins Univ. Press, 1996.

[13] M. Maddah-Ali, S. A. Motahari, and A. K. Khandani, "Signaling over MIMO multibase systems - combination of multiple access and broadcast schemes," in Proc. ISIT, Seattle, WA, Jul. 2006, pp. 2104-2108.

[14] S. Jafar and S. Shamai, "Degrees of freedom for the MIMO $X$ channel," IEEE Trans. Inform. Theory, vol. 54, pp. 151-170, Jan. 2008.

[15] V. Cadambe, S. Jafar, and S. Shamai, "Interference alignment on the deterministic channel and application to fully connected Gaussian interference networks," IEEE Trans. Inf. Theory, vol. 55, pp. 296-274, Jan. 2009.

[16] G. Bresler, A. Parekh, and D. Tse, "The approximate capacity of the many-to-one and one-to-many Gaussian interference channels," IEEE Trans. Inf. Theory, vol. 56, pp. 4566-4592, Sep. 2010.

[17] V. Cadambe, S. A. Jafara, and C. Wang, "Interference alignment with asymmetric complex signaling - settling the Høst-Madsen-Nosratinia conjecture," IEEE Trans. Inf. Theory, vol. 56, pp. 4552-4565, Sep. 2010.

[18] R. Tresch, M. Guillaud, and E. Riegler, "On the achievability of interference alignment in the $K$-user constant MIMO interference channel," in Proc. SSP, Cardiff, Wales, Aug. 2009.

[19] R. Tresch and M. Guillaud, "Celluar interference alignment with imperfect channel knowledge," in Proc. ICC, Dresden, Germany, Jun. 2009.

[20] B. N. Makouei, J. G. Andrews, and R. W. Heath, "A simple SINR characterization for linear interference alignment over uncertain MIMO channels," in Proc. ISIT, Austin, TX, Jun. 2010.

[21] J. Thukral and H. Bölcskei, "Interference alignment with limited feedback," in Proc. ISIT, Seoul, Korea, Jun. 2009.

[22] R. Krishnamachari and M. Varanasi, "Interference alignment under limited feedback for MIMO interference channels," in Proc. ISIT, Austin, TX, Jun. 2010.

[23] B. Nazer, M. Gastpar, S. A. Jafar, and S. Vishwanath, "Ergodic interference alignment," in Proc. ISIT, Seoul, Korea, Jun. 2009.

[24] S. A. Jafar, "Exploriting channel correlations - simple intefererence alignment schemes with no CSIT," in Proc. GLOBECOM, Miami, FL, Dec. 2010.

[25] J. Lee, H. Yu, Y. Sung, and Y. H. Lee, “Adaptive beam tracking for interference alignment for time-varying MIMO interference channels: Conjugate gradient approach," in Proc. ICASSP, Prague, Czech Republic, May 2011.

[26] G. W. Stewart, "An updating algorithm for supspace tracking," IEEE Trans. Signal Process., vol. 40, pp. 1535-1541, Jun. 1992.

[27] C. H. Bischof and G. M. Shroff, "On updating signal subspace," IEEE Trans. Signal Process., vol. 40, pp. 96-105, Jan. 1992.

[28] R. D. DeGroat, "Noniterative subspace tracking," IEEE Trans. Signal Process., vol. 40, pp. 571-577, Mar. 1992.

[29] B. Yang, "Projection approximation subspace tracking," IEEE Trans. Signal Process., vol. 43, pp. 95-107, Jan. 1995.

[30] K. Abed-Meraim, A. Chkeif, and Y. Hua, "Fast orthonormal PAST algorithm,” IEEE Signal Process. Lett., vol. 7, pp. 60-62, Mar. 2000.

[31] C. D. Meyer and G. W. Stewart, "Derivatives and perturbations of eigenvectors," SIAM J. Numer. Anal., vol. 25, pp. 697-691, Jun. 1988.

[32] K. E. Avrachenkov and M. Haviv, "Perturbation of null spaces with application to the eigenvlaue problem and generalized inverses," Linear Algebra and its Appl., vol. 369, pp. 1-25, Aug. 2003.

[33] M. Dong, L. Tong, and B. M. Sadler, "Optimal insertion of pilot symbols for transmissions over time-varying flat fading channels," IEEE Trans. Signal Process., vol. 52, pp. 1403-1418, May 2004.

[34] C. Komninakis, C. Fragouli, A. H. Sayed, and R. D. Wesel, "Multiinput multioutput fading channel tracking and equalization using Kalman estimation," IEEE Trans. Signal Process., vol. 50, pp. 1065-1076, May 2002. 
[35] M. Médard, "The effects upon channel capacity in wireless communications of perfect and imperfect knowledge of the channel," IEEE Trans. Inf. Theory, vol. 46, pp. 933-946, May 2000.

[36] J. Park, Y. Sung, and H. V. Poor, "On Beamformer Design for Multiuser MIMO Interference Channels," 2010 [Online]. Available: ArXiv pre-print cs.IT/1011.6121

[37] D. Popescu and C. Rose, Interference Avoidance Methods for Wireless Systems. New York: Kluwer Academic, 2004

[38] N. Jindal, "MIMO broadcast channels with finite-rate feedback," IEEE Trans. Inf. Theory, vol. 52, pp. 5035-5060, Nov. 2006.

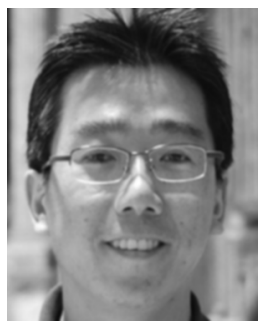

Heejung Yu (S'07) received the B.S. degree in radio science and engineering from the Korea University, Seoul, in 1999, and the M.S. and Ph.D. degrees in electrical engineering from the Korea Advanced Institute of Science and Technology (KAIST), Daejeon, in 2001 and 2011, respectively.

Since 2001, he has been with the Electronics and Telecommunications Research Institute (ETRI), Korea. He has participated in the IEEE 802.11 standardization, where he made technical contributions from 2003. His areas of interest include statistical signal processing and communication theory.

Dr. Yu is the recipient of the Bronze Prize in the 17th Humantech Paper Contest and the Best Paper Award in the 21st Joint Conference on Communications and Information (JCCI) in 2011.

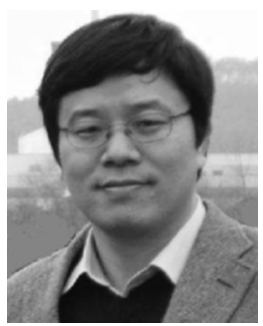

Youngchul Sung (S'92-M'93-SM'09) received B.S. and M.S. degrees from Seoul National University, Seoul, Korea, in electronics engineering in 1993 and 1995, respectively, and the Ph.D. degree in electrical and computer engineering from Cornell University, Ithaca NY, in 2005.

$\mathrm{He}$ is an Associate Professor in the Department of Electrical Engineering, Korea Advanced Institute of Science and Technology (KAIST), Daejeon. From 2005 until 2007, he was a senior engineer in the Corporate R\&D Center, Qualcomm, Inc., San Diego, $\mathrm{CA}$, and also worked as a senior research engineer with LG Electronics, Ltd.,
Seoul, from 1995 to 2000 . His research interests include signal processing for communications, statistical signal processing, and asymptotic statistics with applications to wireless communications and related areas.

Dr. Sung is an associate member of the IEEE SPS SPCOM TC, a member of Signal and Information Processing Theory and Methods (SIPTM) TC of Asia-Pacific Signal and Information Processing Association (APSIPA), a TPC member of Globecom 2011/2010/2009, ICC 2011, MILCOM 2010, DCOSS 2010, WiOpt 2009, and its sponsorship chair, APSIPA 2010/2009, IEEE SAM 2008 .

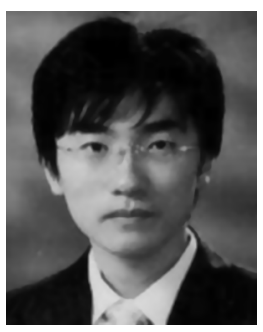

Haksoo Kim (S'06) received the B.S. and M.S. degrees in electrical engineering for Korea Advanced Institute of Science and Technology (KAIST), Daejeon, in 2006 and 2008, respectively.

$\mathrm{He}$ is currently working toward the Ph.D. degree in the Department of Electrical Engineering, KAIST. His research interests include statistical signal processing and communication theory.

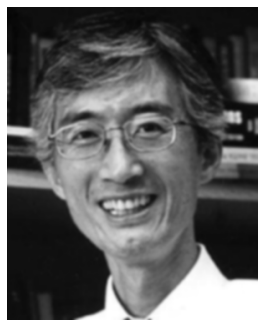

Yong H. Lee (S'81-M'84-SM'98) was born in Seoul, Korea, on July 12, 1955. He received the B.S. and M.S. degrees in electrical engineering from Seoul National University, in 1978 and 1980 respectively, and the Ph.D. degree in electrical engineering from the University of Pennsylvania, Philadelphia, in 1984.

From 1984 to 1988, he was an Assistant Professor with the Department of Electrical and Computer Engineering, State University of New York, Buffalo. Since 1989, he has been with the Department of Electrical Engineering, Korea Advanced Institute of Science and Technology (KAIST), where he is currently a Professor and the Provost of KAIST. His research activities are in the area of communication signal processing, which includes interference management, resource allocation, synchronization, and estimation for OFDM/MIMO systems with relays. 